\title{
LOW CASE NUMBERS ENABLE LONG-TERM STABLE PANDEMIC CONTROL WITHOUT LOCKDOWNS
}

\author{
Sebastian Contreras ${ }^{1}$, Jonas Dehning ${ }^{1}$, Sebastian B. Mohr ${ }^{1}$, F. Paul Spitzner ${ }^{1}$, and Viola \\ Priesemann $^{1,2^{*}}$ \\ ${ }^{1}$ Max Planck Institute for Dynamics and Self-Organization, Am Faßberg 17, 37077 Göttingen, Germany. \\ ${ }^{2}$ Department of Physics, University of Göttingen, Friedrich-Hund-Platz 1, 37077 Göttingen, Germany. \\ All authors contributed equally.
}

\begin{abstract}
The traditional long-term solutions for epidemic control involve eradication or herd immunity ${ }^{\text {[;: }}$. Neither of them will be attained within a few months for the COVID-19 pandemic. Here, we analytically derive the existence of a third, viable solution: a stable equilibrium at low case numbers, where test-trace-and-isolate policies partially compensate for local spreading events, and only moderate contact restrictions remain necessary. Across wide parameter ranges of our complementary compartmental model ${ }^{\mathbf{3}}$, the equilibrium is reached at or below 10 daily new cases per million people. Such low levels had been maintained over months in most European countries. However, this equilibrium is endangered (i) if contact restrictions are relaxed, or (ii) if case numbers grow too high. The latter destabilisation marks a novel tipping point beyond which the spread self-accelerates because test-trace-and-isolate capacities are overwhelmed. To reestablish control quickly, a lockdown is required. We show that a lockdown is either effective within a few weeks, or tends to fail its aim. If effective, recurring lockdowns are not necessary - contrary to the oscillating dynamics previously presented in the context of circuit breakers ${ }^{\text {; } ; ; ; ; ;}$, and contrary to a regime with high case numbers - if moderate contact reductions are maintained. Hence, at low case numbers, the control is easier, and more freedom can be granted. We demonstrate that this strategy reduces case numbers and fatalities by a factor of 5 compared to a strategy focused only on avoiding major congestion of hospitals. Furthermore, our solution minimises lockdown duration, and hence economic impact. In the long term, control will successively become easier due to immunity through vaccination or large scale testing programmes. International coordination would facilitate even more the implementation of this solution.
\end{abstract}

As SARS-CoV-2 is becoming endemic and knowledge about its spreading is accumulated, it becomes clear that neither global eradication nor herd immunity will be achieved soon. Eradication is hindered by the worldwide prevalence and by asymptomatic spreading. Reaching herd immunity without an effective vaccine or medication would take several years and cost countless deaths, especially among the elderly $\mathbb{\square} ; \mathbb{\square}$. Moreover,

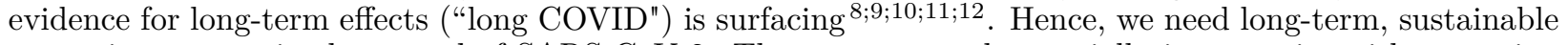
strategies to contain the spread of SARS-CoV-2. The common goal, especially in countries with an ageing population, should be to minimise the number of infections and, thereby, allow reliable planning for individuals and the economy - while not constraining individuals' number of contacts too much. Intuitively, a regime with low case numbers would benefit not only public health and psychological well-being but would also profit the economy ${ }^{\amalg 3 ; \square]}$.

However, control of SARS-CoV-2 is challenging. Many infections originate from asymptomatic or pre-

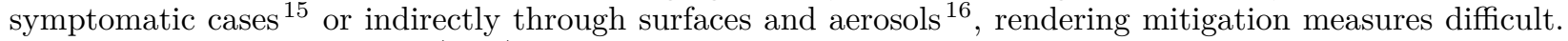
Within test-trace-and-isolate (TTI) strategies, the contribution of purely symptom-driven testing is limited, but together with contact tracing, it can uncover asymptomatic chains of infections. Additional challenges

\footnotetext{
*viola.priesemann@ds.mpg.de
} 
medRxiv preprint doi: https://doi.org/10.1101/2020.12.10.20247023; this version posted December 11, 2020. The copyright holder for this preprint (which was not certified by peer review) is the author/funder, who has granted medRxiv a license to display the preprint in perpetuity. All rights reserved. No reuse allowed without permission.

Low case numbers enable long-term stable pandemic control without lockdowns

are the potential influx of SARS-CoV-2 infections (brought in by travellers or commuting workers from abroad), imperfect quarantine, limited compliance, and delays in TTI and case-reporting. Lastly, any countries capacity to perform TTI is limited, so that spreading dynamics change depending on the level of case numbers. Understanding these dynamics is crucial for informed policy decisions.

\section{Analytical framework: Overview}

We analytically show the existence of a stable regime at low case numbers, where control of SARS-CoV-2 is much easier to achieve and sustain. In addition, we investigate mitigation strategies and long term control for COVID-19, where we build on our past work to understand the effectiveness of non-pharmaceutical interventions, particularly test-trace-and-isolate (TTI) strategies ${ }^{[3 ;[\square] ;[1]}$. For quantitative assessments, we adapt an SEIR-type compartmental model ${ }^{\mathbb{1 U}}$ to explicitly include a realistic TTI system that considers the challenges above.

A central parameter for our analysis is the effective reduction of contagious contacts $k_{t}$ (relative to preCOVID-19). More precisely, $k_{t}$ does not simply refer to the reduction of contacts a person has, but rather to the encounters that bear a potential for transmission. Apart from direct contact reduction, contributions to $k_{t}$ also come from improved hygiene, mandatory face-mask policies, frequent ventilation of closed spaces, and avoiding indoor gatherings, among other precautionary measures. As the latter measures are relatively fixed, direct contact reduction remains the central free variable, which is also the one tuned during lockdowns. All other parameters (and their references) are listed in Table $\mathbf{5 7}$.

\section{Equilibrium at low case numbers}

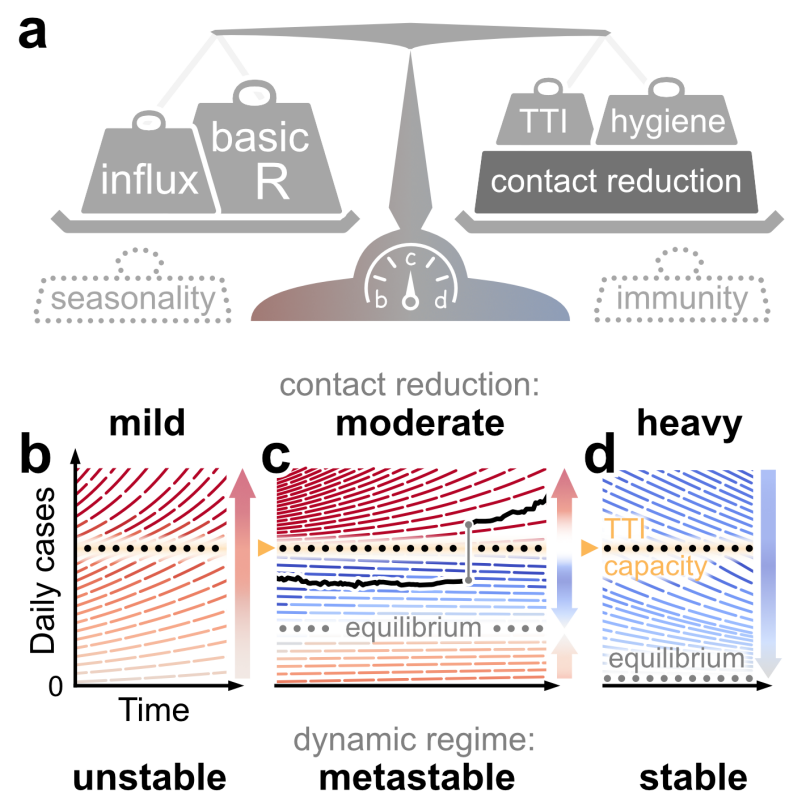

Figure 1: Spreading dynamics depend not only on the balance between destabilising and stabilising contributions, but also on the level of case numbers itself. a: Among the factors that destabilise the spread, we find the basic reproduction number $R_{0}$ and the external influx of infections (and possibly seasonality). On the other hand, various contributions can stabilise it, including increased hygiene, test-trace-isolate (TTI) strategies, contact reduction, but also immunity. We specifically investigated how contact reduction $k_{t}$ and limited TTI capacity determine the stabilisation of case numbers. b: Assuming only mild contact reduction $\left(k_{t}=20 \%\right.$ compared to preCOVID-19 times), TTI is not sufficient to prevent increasing case numbers - even when TTI capacity is still available; case numbers are increasing. c: At moderate contact reduction $\left(k_{t}=40 \%\right.$ ), a metastable equilibrium emerges (grey dots) to which case numbers converge - if case numbers do not exceed the TTI capacity. However, destabilizing events (as, e.g., a sudden influx of infections), can push a previously stable system above the TTI capacity and lead to an uncontrolled spread (black line as an example). d: Assuming heavy contact reduction $\left(k_{t}=60 \%\right)$, case numbers decrease even if the TTI capacity is exceeded. 
Between the scenarios of eradication of the disease or uncontrolled spreading, we find a regime where the spread reaches an equilibrium at low daily case numbers. The main control parameter that determines whether the system can reach an equilibrium is the contact reduction $k_{t}$.

If the contact reduction $k_{t}$ is mild, case numbers grow exponentially, as measures could not counterbalance the basic reproduction number $\left(R_{0} \approx 3.3\right.$ for SARS-CoV-2 2 ; $\left.; 21 ; 22\right)$ (Fig. $\left.\square \mathrm{b}\right)$. In contrast, if the contact reduction is strong and (together with hygiene and TTI) outweighs the drive by the basic reproduction number, case numbers decrease to a low equilibrium value (Fig. Đd).

Importantly, if the contact reduction is moderate (and just-about balances the drive by the basic reproduction number), we find a metastable regime: The spread is stabilised if and only if the overall case numbers are sufficiently low to enable fast and efficient TTI (Fig. Mc). However, this control is lost if the limited TTI capacity is overwhelmed. Beyond that tipping point, the number of cases starts to grow exponentially as increasingly more infectious individuals remain undetected ${ }^{\text {[3. }}$.

The capacity of TTI determines the minimal required contact reduction for controlling case numbers around an equilibrium. If case numbers are sufficiently below the TTI capacity limit, the required contact reduction to maintain the (meta-)stable regime is only $k_{t}^{\text {crit }}=39 \%$ (95\% confidence interval (CI):[24, 53]). However, if case numbers exceed the TTI capacity limit, a considerably stronger contact reduction of $k_{t}^{\text {crit }}=58 \%(95 \%$ CI: $[53,62]$ ) is required to reach the stable regime (Fig. ㄱ b, Fig. \$4 and Table 5.3).

\section{Equilibrium depends on influx and contact reduction}
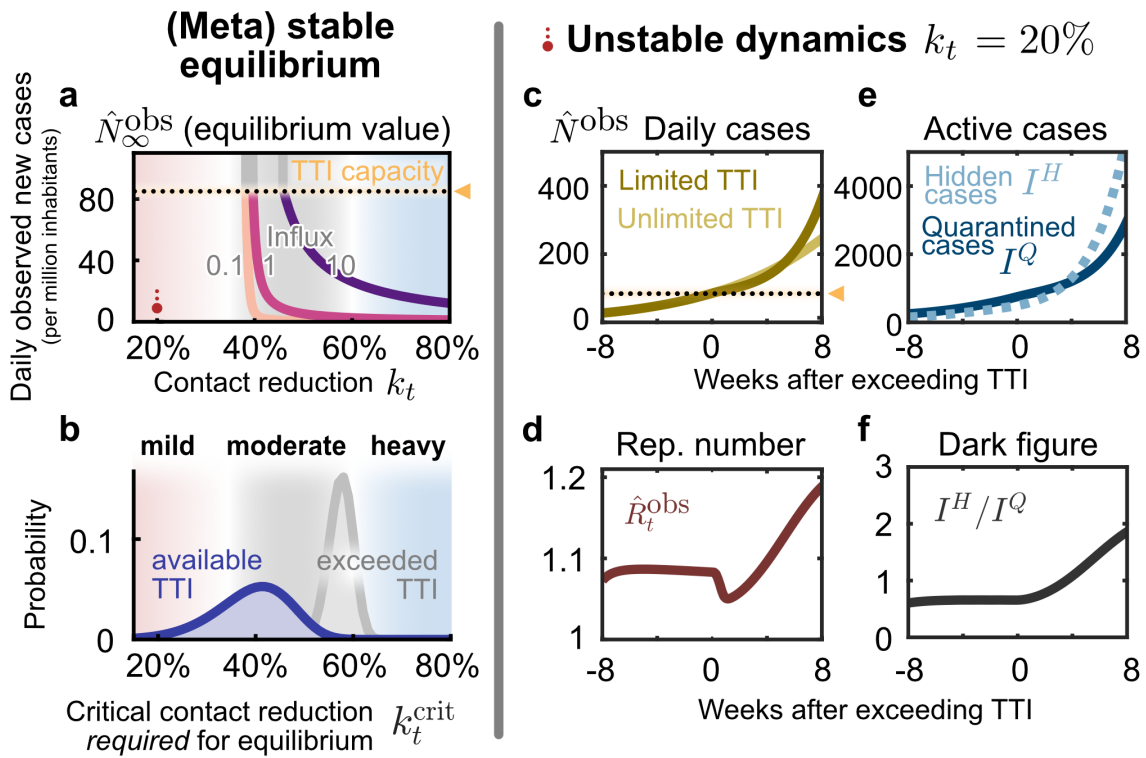

Figure 2: (a, b) In the stable and metastable regimes, daily new cases approach an equilibrium value $\hat{N}_{\infty}^{\text {obs }}$ that depends on contact reduction $k_{t}$ and external influx of new cases $\Phi_{t}$. a: The equilibrium value $\hat{N}_{\infty}^{\text {obs }}$ increases with weaker contact reduction $k_{t}$ or higher influx $\Phi_{t}$. No equilibrium is reached, if either $k_{t}$ or $\Phi_{t}$ are above the respective (critical) threshold values. b: The critical value $k_{t}^{\text {crit }}$ represents the minimal contact reduction that is required to reach equilibrium and stabilise case numbers. If case numbers are below the TTI capacity limit, lower values of $k_{t}^{\text {crit }}$ are required for stabilisation (blue) than if cases exceed TTI (grey). Confidence intervals originate from error propagation of the uncertainty of the underlying model parameters. (c-f) In the unstable dynamic regime $\left(k_{t}=20 \%\right)$, a tipping point is visible when exceeding TTI capacity. We observe a self-accelerating increase of case numbers after crossing the TTI limit (c) and a subsequent increase of the reproduction number (d). Furthermore, the absolute number (e) and the proportion — "dark figure" (f) — of cases that remain unnoticed increase over time.

If an equilibrium is reached, the precise value of daily new cases $\hat{N}_{\infty}^{\text {obs }}$ at which the system stabilises depends on both the reduction of contagious contacts $k_{t}$ and the external influx of new cases $\Phi_{t}$ (Fig. Zla). In general, for realistic low values of influx $\Phi_{t}$, the equilibrium level $\hat{N}_{\infty}^{\text {obs }}$ is low. However, $\hat{N}_{\infty}^{\text {obs }}$ increases steeply (diverges) when the contact reduction $k_{t}$ approaches the tipping point to unstable dynamics (Fig. ㅍa,b). 
Such a divergence near a critical point $k_{t}^{\text {crit }}$ is a general feature of continuous transitions between stable and unstable dynamics ${ }^{\text {[3;; } ; 4]}$. As a rule of thumb, in an analytical mean-field approximation, $\hat{N}_{\infty}^{\text {obs }}$ would be proportional to $\Phi_{t}$ and diverge when $k_{t}$ approaches its critical value $k_{t}^{\text {crit }}$ from above: $\hat{N}_{\infty}^{\text {obs }} \propto \Phi_{t} /\left(k_{t}-k_{t}^{\text {crit }}\right)^{\text {四 }}$. Robust control of the pandemic requires maintaining a sufficient safety-margin from the tipping point (and the subsequent transition to instability) for two reasons. First, small fluctuations in $k_{t}$, $\Phi_{t}$ (or other model variables) could easily destabilise the system. Second, near the critical value $k_{t}^{\text {crit }}$, reductions in $k_{t}$ are especially effective: already small further reductions below $k_{t}^{\text {crit }}$ lead to significantly lower stable case numbers (Fig. Zla). Already with moderate contact reduction $\left(40 \%<k_{t}<50 \%\right)$, the spread can be stabilised to a regime of case numbers clearly below 10 per million (Fig. $\mathbf{5 1}$ b, lower right region).

\section{Limited TTI and self-acceleration}

If mitigation measures are insufficient, case numbers rise and eventually surpass the TTI capacity limit. Beyond it, health authorities are not able to efficiently trace contacts and uncover infection chains, thus the control of the spread becomes more difficult. We start our scenario with a slight increase in case numbers over a few months, as seen in many European countries throughout the summer 2020 (Fig. \$5.5 and Fig. \$6). A tipping point is then visible in the following observables (Fig. 『 $\mathrm{c}-\mathrm{f}$ ):

First, when case numbers surpass the TTI capacity, the increase in daily new observed cases $\hat{N}^{\text {obs }}$ becomes steeper, growing even faster than the previous exponential growth (Fig. [ c, full versus faint line). The spread self-accelerates because increasingly more contacts are missed, which, in turn, infect more people. Importantly, in this scenario the accelerated spread arises solely because of exceeding the TTI limit without any underlying behaviour change among the population.

Second, after case numbers surpass the TTI limit, the observed reproduction number $\hat{R}_{t}^{\text {obs }}$, which had been only slightly above the critical value of unity, increases significantly by about $20 \%$ (Fig. Ud). This reflects a gradual loss of control over the spread and explains the faster-than-exponential growth of case numbers. The initial dip in $\hat{R}_{t}^{\text {obs }}$ is a side-effect of the limited testing: As increasingly many cases are missed, the observed reproduction number reduces transiently.

Third, compared to the infectious individuals who are quarantined $I^{Q}$, the number of infectious individuals who are hidden $I^{H}$ (i.e. those who are not isolated or in quarantine) increases disproportionately (Fig. $\nabla \mathrm{e}$ ) which is measured by the "dark figure" $\left(I^{H} / I^{Q}\right)$ (Fig. $\nabla \mathrm{f}$ ). The hidden infectious individuals are the silent drivers of the spread as they, unaware of being infectious, inadvertently transmit the virus. This implies a considerable risk, especially for vulnerable people. At low case numbers, the TTI system is capable to compensate the hidden spread, because it uncovers hidden cases through contact tracing. However, at high case numbers, the TTI becomes inefficient: If the TTI measures are "slower than the viral spread", many contacts cannot be quarantined before they become spreaders.

\section{Re-establishing control with lockdowns}

Once the number of new infections has overwhelmed the TTI system, re-establishing control can be challenging. A recent suggestion is the application of a circuit breaker ${ }^{\text {a; } ; 5 ; 0}$, a short lockdown to significantly lower the number of daily new infections. Already during the first wave, lockdowns have proven capable to lower case numbers by a factor 2 or more every week (corresponding to an observed reproduction number of $\left.\hat{R}_{t}^{\text {obs }} \approx 0.7\right)$. With the knowledge we now have acquired about the spreading of SARS-CoV-2, more targeted restrictions may yield a similarly strong effect.

Inspired by the lockdowns installed in many countries ${ }^{[2.5}$, we assume a default lockdown of four weeks, starting four weeks after case numbers exceed the TTI capacity limit, and a strong reduction of contagious contacts of $k_{\mathrm{LD}}=75 \%$ (which corresponds to an $\hat{R}_{t}^{\text {eff }} \approx 0.85$, see Table 54 ). We further assume that during lockdown the external influx of infections $\Phi_{t}$ is reduced by a factor ten, and that after the lockdown, a moderate contact reduction $\left(k_{\mathrm{nLD}}=40 \%\right)$ is maintained. By varying the parameters of this default lockdown, we show in the following that the lockdown strength, duration, and starting time determine whether the lockdown succeeds or fails to reach equilibrium.

In our scenario, a lockdown duration of four weeks is sufficient to reach the stable regime (Fig. B a). However, if lifted too early (before completing four weeks), then case numbers will rise again shortly after. The shorter an insufficient lockdown, the faster case numbers will rise again. Also, it is advantageous to remain in 


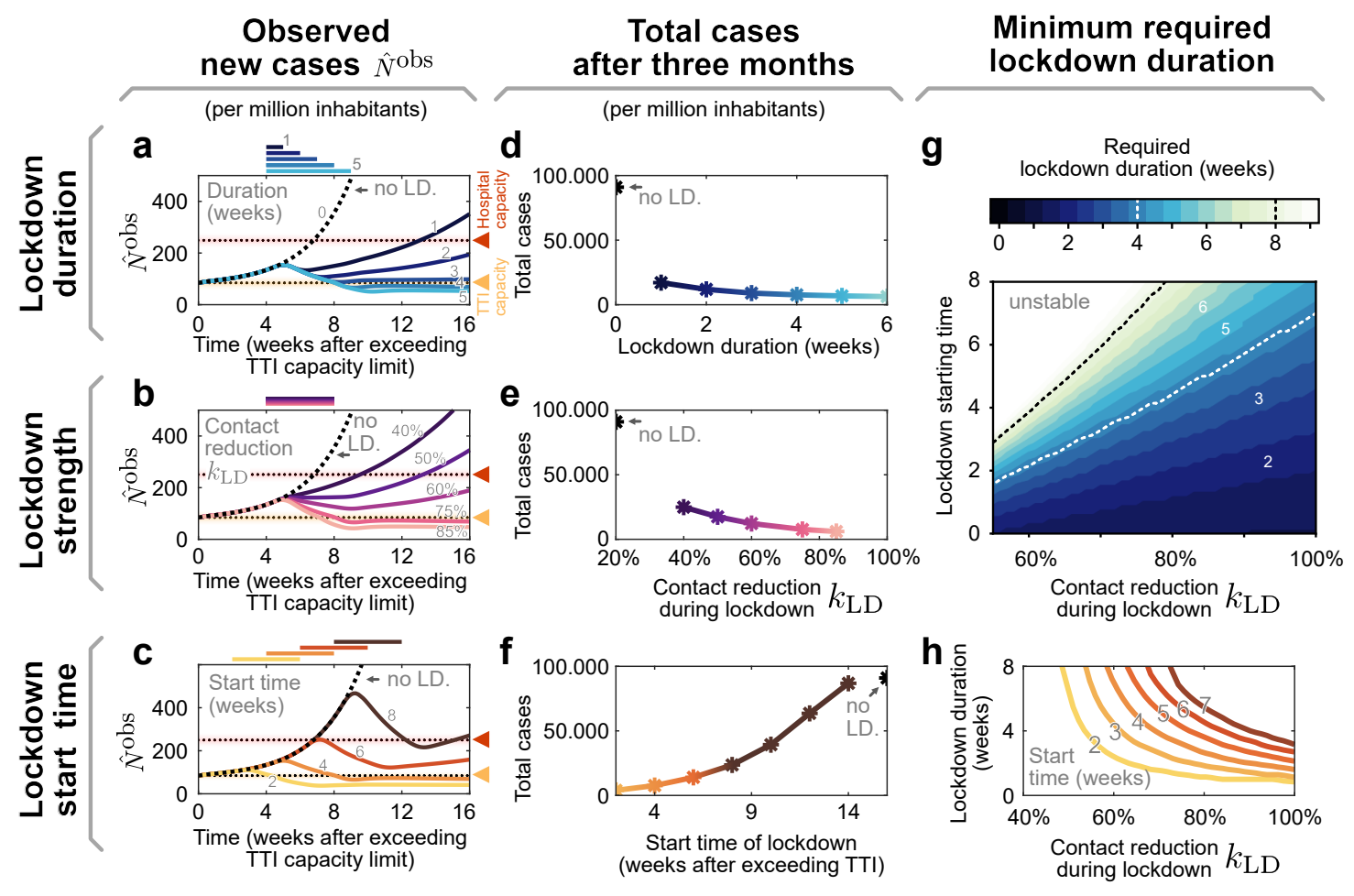

Figure 3: The effectiveness of a lockdown depends on three main parameters: its duration, stringency (strength), and starting time. (a-c) Observed daily new cases for a lockdown (abbreviated as LD) which is enacted after the TTI capacity has been exceeded. Reference parameters are a lockdown duration of 4 weeks, contact reduction during lockdown of $k_{\mathrm{LD}}=75 \%$ and a start time at 4 weeks after exceeding TTI capacity. We vary lockdown duration (a), lockdown strength (b) and lockdown starting time (c) to investigate whether stable case numbers can be reached. (d-f) Total cases after three months, if the lockdown is parameterised as described in panels a-c, respectively. (g, h:) The minimal required duration of lockdown to reach equilibrium depends both on strength and start time. g: Heavy contact reduction and timely lockdown enacting can create effective short lockdowns $(\leq 2$ weeks, lower left, dark region). Whereas with mild contact reduction and very late start times, lockdowns become ineffective even when they last indefinitely (Upper left, bright region). h: Horizontal slices through the colour-map (g). Here, colours match panels $(\mathbf{c}, \mathbf{f})$ and correspond to the lockdown start time.

lockdown for a short time even after case number have fallen below the TTI limit — in order to establish a safety-margin, as shown above. Overall, the major challenge is not to ease the lockdown too early, as otherwise the earlier success is soon squandered.

During lockdown, it is necessary to reduce contagious contacts $k_{t}$ severely in order to decrease case numbers below the TTI capacity limit (Fig. [ B b). In our scenario, the contacts have to be reduced by at least $k_{\mathrm{LD}}=75 \%$ to bring the system back to equilibrium. A lockdown that is only slightly weaker cannot reverse the spread to a decline of cases. Furthermore, increasing the lockdown strength decreases both the required lockdown duration (Fig. B g,h) and the total number of cases accumulated over three months (Fig. B e). This shows that stricter lockdowns imply shorter-lasting social and economical restrictions.

The earlier a lockdown begins after exceeding the TTI capacity limit, the faster control can be re-established and constraints can be loosened again (Fig. B c). If started right after crossing the threshold, in principle, only a few days of lockdown are necessary to bring back case numbers below TTI capacity limit. On the other hand, if the lockdown is started weeks later, its duration needs to increase (Fig. B c,d) and the total number of cases will be significantly larger (Fig. B] f).

In conclusion, to re-establish control, a lockdown needs to be strong enough to reach equilibrium within a few weeks, or it fails almost completely.

The parameter regime between these two options is quite narrow; it is not likely that equilibrium can eventually be reached as a lockdown exceeds many weeks (cf. Fig. [ $\mathrm{l}$ h). For practical policies, this means 
Low case numbers enable long-term stable pandemic control without lockdowns

that if a lockdown does not start to show clear effects after 2 or 3 weeks, then the strategy should be revised (this assessment time is necessary due to the delay of 1-2 weeks between contagion and case-report).

\section{Maintaining control without lockdowns}

We show that repeated lockdowns are not required to maintain control over the COVID-19 spread if moderate contact reduction is maintained once case numbers are below the TTI capacity limit (an initial lockdown might still be necessary to establish control). A natural goal would be to keep case numbers below the hospital capacity. However, our model suggests that lowering them below TTI capacity requires less contact restrictions (in the long-term), involves a shorter total lockdown duration, and costs less lives. In the following we compare the long-term perspective of these two goals and their dependence on the necessary contact reduction.
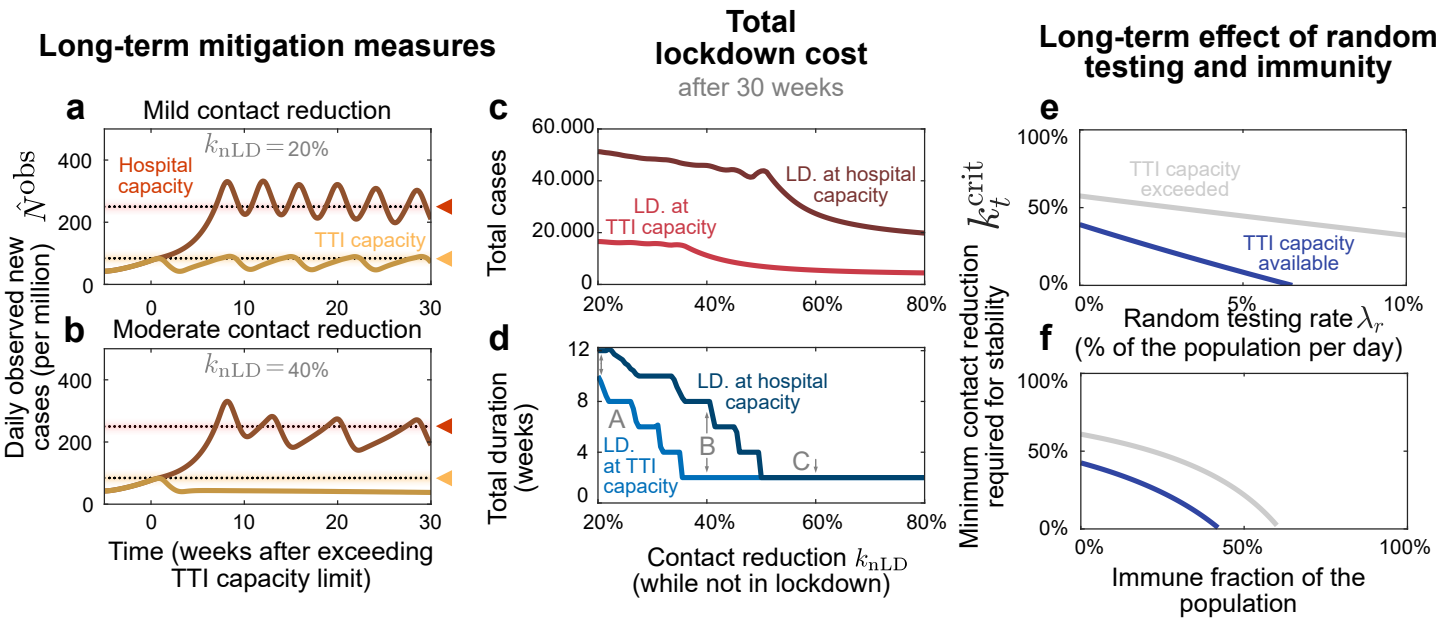

Figure 4: On a long-term perspective, recurrent lockdowns are not required if the subsequent contact reduction $k_{\mathrm{nLD}}$ is sufficient to reach equilibrium. A two-week lockdown of default strength $\left(k_{\mathrm{LD}}=75 \%\right)$ is either enacted when the test-trace-and-isolate (TTI, yellow lines) or the hospital (red lines) capacity limits are crossed. a: Given only a mild contact reduction while not in lockdown $k_{\mathrm{nLD}}=20 \%$, the imposed lockdowns need to reoccur with a high frequency. Moreover, the frequency at which they reoccur is higher at high case numbers (aiming at hospital capacity) than when aiming at the TTI capacity limit because of the self-accelerating effect described previously. b: If the contact reduction remains moderate $k_{\mathrm{nLD}}=40 \%$, lockdowns only need to reoccur if they fail to bring case numbers below TTI capacity. c, d: The total cost (in terms of cumulative cases and total lockdown duration) depends on the level of contact reduction and the lockdown policy: The cost is low if lockdowns are initiated at the TTI capacity limit and if $k_{\mathrm{nLD}}$ is high. e, f: In the future, the minimal contact reduction required for stability $k_{t}^{\text {crit }}$ may be lowered by the implementation of large-scale random testing (e) or by immunisation of the population $(\mathbf{f})$.

In our scenario (Fig. 四), we start from the unstable regime, where the initial contact reduction $k_{t}=20 \%$ is not sufficient to control the spread. We start a two-week lockdown when crossing either the TTI or the hospital capacity. During the lockdown, contacts are reduced by $k_{\mathrm{LD}}=75 \%$. After the first and all subsequent lockdowns, contacts $k_{\mathrm{nLD}}$ are reduced by $20 \%$ or $40 \%$ relative to pre-COVID-19 levels, representing mild or moderate reduction. When assuming mild contact reduction after lockdowns, case numbers rise after lifting the lockdown, independent of the chosen threshold (TTI or hospital capacity, Fig. 目 a). Thus, repeated lockdowns are necessary.

However, maintaining a moderate contact reduction while not in lockdown $\left(k_{\mathrm{nLD}}=40 \%\right)$ is sufficient to stay within the metastable regime - if lockdowns are enacted such that case numbers stay below the TTI capacity (Fig. W b, yellow line). This is a promising perspective for a long-term control strategy that avoids recurrent lockdowns. Otherwise, if case numbers are above TTI capacity limit but below hospital capacity, a control of the pandemic requires repeated lockdowns (Fig. $\mathbb{b} \mathrm{b}$, red line) or stronger contact reductions.

The advantage of the strategy to stay below the TTI capacity limit becomes very clear when considering the total cost of the required lockdowns: Independently of the level of contact reduction, (i) the total number of cases (and consequently deaths and "long-COVID" risk) is lower (Fig. (⿴囗十), (ii) the total duration spent

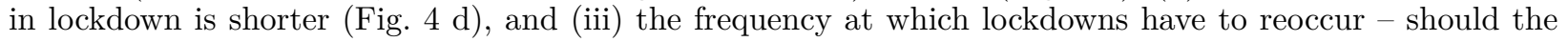


Low case numbers enable long-term stable pandemic control without lockdowns

after-lockdown contact reduction not be enough to grant metastability - is lower (Fig. 团 a). As case numbers and lockdown duration indicate economic costs, a strategy that respects the TTI limit offers a low economic toll, enables mid-term planning and provides trust to people and society.

In the future, large-scale testing ${ }^{[26 ; 27 ;[2]}$ and immunisation (either through vaccination or lasting post-infection immunity) will play a role for long-term strategies. Both will reduce the necessary reduction of contacts to maintain control (Fig. 四 e, f). Early effects of immunity can be seen in our scenario of the system that is stabilised at hospital capacity: The need for lockdowns becomes less frequent over time (Fig. 团a, b). However, acquiring natural immunity comes at the cost of a prolonged high level of case numbers, subsequent "long COVID" cases ${ }^{\mathrm{Q} ;[\Pi ; \square ; \square]}$ and deceased people. Whereas, the duration of the immunity is not known yet ${ }^{[\mathrm{ZI}}$, this phenomenon still shows that immunity effects play an increasing role as model predictions extend further into the future.

\section{Discussion}

We demonstrated that between the two extremes of eradication and uncontrolled spread, a metastable regime of SARS-CoV2 spreading exist. In such a regime, every person only has to reduce their contacts moderately, while case numbers can still be maintained robustly at low levels, because the test-trace-and-isolate (TTI) system can operate efficiently. If this regime is within reach, keeping case numbers below TTI capacity is a suitable strategy for the control of COVID-19 that features low fatalities and a small societal burden.

Among countries around the world, the wide range and great variability in the levels of case numbers illustrate that governmental policies and the chosen strategy to face COVID-19 have great impact (see Supplementary Notes 2.1). Sustained high levels of more than 100 daily new cases per million have been observed in several (but not exclusively) American countries (Supp. Fig. 5.3 a). This shows that high levels of daily new infections can be maintained. However, the stringency of interventions is similar to other countries ${ }^{130}$, and the prospect of reaching herd immunity is still far away. On the other hand, very low case numbers and even local eradication have been achieved by several South- and East-Asian countries, Australia and New-Zealand. At the time of writing, these countries profit from the absorbing state at zero SARS-CoV-2 infections, but maintaining this state requires strong international travel restrictions (Supp. Fig. \$3 c). An intermediate level of case numbers could predominantly be observed over the summer 2020 in Europe. Case numbers for many countries were typically around 10 daily new cases per million (Supp. Fig. \$.3 b), even though contacts were only mildly restricted. These stable numbers demonstrate that also in practice a regime below TTI capacity limits is maintainable. Nonetheless, in September the spread significantly accelerated in a number of European countries, when case numbers began to exceed 20 to 50 daily new cases per million (Fig. 56). Beyond these levels of case numbers, the TTI systems began to be overwhelmed, making a control difficult - in line with the results of our model.

In order to focus our model on the general spreading dynamics, we made simplifying assumptions: We assumed that spreading happens homogeneously in the population, with neither regional nor age-related differences. In reality, heterogeneous spreading can lead to regionally differing case numbers, which illustrates the need for regional monitoring of the remaining TTI capacity to allow for early and targeted control measures. In our scenarios, we further assumed that the behaviour of the population and subsequent contact reduction is constant over time (except during lockdown). Real situations are more dynamic, necessitating frequent reevaluations of the current restrictions and mitigation measures. We also assumed a constant effectiveness of TTI if below the capacity limit, but if case numbers are very low, all the available test- and trace-efforts could be concentrated on the remaining infection chains. This would further facilitate a control at low case numbers. Overall, our analytical results describe the general behaviour across countries well and identify the relevant factors for the control of the pandemic.

Quantitatively, our assumptions regarding the efficiency of TTI are in agreement with those of other modelling studies. Agent-based models with detailed contact structures ${ }^{\text {15;;32 }}$ and mean-field models ${ }^{33 ; ; 34 ; 33 ; ; 36}$ both agree that TTI measures are an important contribution for the control of the pandemic but typically do not suffice alone. Their success strongly depends on their implementation: Fast testing, rigorous isolation, and a large proportion of traced contacts are essential. Given our informed assumptions about these parameters, our model shows that TTI can only compensate a basic reproduction number $R_{0}$ of 3.3 - if contagious contacts are also reduced by about $40 \%$ (95\% CI: [24,53]). This is in agreement with the results of other studies ${ }^{37 ; ; 34 ; ; 38 ; 30 ; ; 32}$.

The capacity limit of TTI plays a central role to inform policymakers when to issue preventive measures. The precise limit of TTI depends on a number of factors, including the number of available tests, personnel at 
Low case numbers enable long-term stable pandemic control without lockdowns

the tracing units, potentially a tracing app ${ }^{[4]}$, and the number of relevant contacts a person has on average. Already the latter can easily differ by a factor 10, depending on contact restrictions and cultural factors 139 . For our scenarios, we assumed the capacity is reached at about 100 daily new cases per million, which is comparably high. Independent of the exact value, when this limit is approached, the risk of tipping over to uncontrolled spread strongly increases, and countermeasures should be taken without delay. Otherwise, the spread might self-accelerate and get out of control.

Our results show that a stable equilibrium at low case numbers can be maintained with a moderate contact reduction of about $40 \%$ less contagious contacts compared to pre-COVID-19. This level of contact reduction can be achieved with preventive mitigation measures, as shown by studies analysing the effectiveness of nonpharmaceutical interventions during the first wave ${ }^{40 ; ; 41 ; ;[1 ; ;[2]}$. Restrictions on the maximum size of gatherings already lead to an effective reduction in the range of 10-40\% $100 ; 20 ; ; 118$. Improved hygiene, frequent ventilation of rooms, and the compulsory use of masks can further reduce the number of infectious contacts (by a factor that is more difficult to estimate $\left.{ }^{42 ; 73.3}\right)$. In conclusion, until a vaccination or better treatment is developed, the regime of low case numbers is very promising for a mid- and long-term management of the pandemic, as it poses the least burden on economy and society.

On the other hand, stabilising the spread at higher levels of case numbers (e.g. at the hospital capacity limit) requires more stringent and more frequent non-pharmaceutical interventions, because the TTI system cannot operate efficiently. Examples of more stringent measures are the closure of schools and public businesses, stay-at-home orders, and contact ban policies ${ }^{40 ;} ; 41 ; 107$.

Concluding, the recommendation from this paper is to reach and maintain low case numbers that allow an efficient operation of TTI measures. To this end, it is mandatory to counteract local super-spreading events (or an acute influx of infections) as early as possible and to sustain a sufficient level of mitigation measures. If low case numbers are reached and maintained throughout Europe, or even internationally, then travel and commute could take place almost unrestricted; every country would profit from the stability of its neighbours.

\section{References of main text (1-44) and supplementary (45-70)}

[1] Randolph, H. E. \& Barreiro, L. B. Herd immunity: Understanding covid-19. Immunity 52, 737-741 (2020).

[2] Heywood, A. E. \& Macintyre, C. R. Elimination of covid-19: what would it look like and is it possible? The Lancet Infectious Diseases 20, 1005-1007 (2020).

[3] Contreras, S. et al. The challenges of containing sars-cov-2 via test-trace-and-isolate. arXiv preprint arXiv:2009.05732 (2020).

[4] Keeling, M. J. et al. Precautionary breaks: planned, limited duration circuit breaks to control the prevalence of COVID-19. medRxiv 2020.10.13.20211813 (2020). URL https://www.medrxiv.org/ content/10.1101/2020.10.13.20211813v1.

[5] Mahase, E. Covid-19: Experts recommend two week circuit break to reduce transmission in the UK. BMJ 371 (2020). URL https://wwW.bmj.com/content/371/bmj.m4038.

[6] Kmietowicz, Z. Covid-19: There is no alternative, says Johnson, announcing new restrictions for England. BMJ 371 (2020). URL https://www. bmj.com/content/371/bmj.m4247.

[7] Verity, R. et al. Estimates of the severity of coronavirus disease 2019: a model-based analysis. The Lancet infectious diseases (2020).

[8] Alwan, N. A. et al. Scientific consensus on the COVID-19 pandemic: we need to act now. The Lancet 6736, 19-20 (2020).

[9] Fraser, E. Long term respiratory complications of covid-19. BMJ 370 (2020). URL https://www.bmj . com/content/370/bmj.m3001. https://www. bmj.com/content/370/bmj.m3001.full.pdf.

[10] Rubin, R. As Their Numbers Grow, COVID-19 Long Haulers Stump Experts. JAMA 324, 1381 (2020). URL https://jamanetwork.com/journals/jama/fullarticle/2771111.

[11] Greenhalgh, T., Knight, M., ACourt, C., Buxton, M. \& Husain, L. Management of post-acute covid-19 in primary care. BMJ $\mathbf{3 7 0}$ (2020). URL https://www.bmj.com/content/370/bmj.m3026.

[12] Topol, E. J. COVID-19 can affect the heart. Science 370, 408-409 (2020). URL https://science. sciencemag.org/content/370/6515/408. 
medRxiv preprint doi: https://doi.org/10.1101/2020.12.10.20247023; this version posted December 11, 2020. The copyright holder for this preprint (which was not certified by peer review) is the author/funder, who has granted medRxiv a license to display the preprint in perpetuity. All rights reserved. No reuse allowed without permission.

Low case numbers enable long-term stable pandemic control without lockdowns

[13] Scherbina, A. Determining the optimal duration of the covid-19 suppression policy: A cost-benefit analysis. Economics Working Paper 3 (2020).

[14] Lin, Z. \& Meissner, C. M. Health vs. wealth? public health policies and the economy during covid-19. Public Health Policies and the Economy During Covid-19 (May 2020). NBER Working Paper (2020).

[15] Li, R. et al. Substantial undocumented infection facilitates the rapid dissemination of novel coronavirus (SARS-CoV-2). Science 368, 489-493 (2020).

[16] Van Doremalen, N. et al. Aerosol and surface stability of sars-cov-2 as compared with sars-cov-1. New England Journal of Medicine 382, 1564-1567 (2020).

[17] Linden, M. et al. Case numbers beyond contact tracing capacity are endangering the containment of COVID-19. Dtsch Arztebl International 117, 790-791 (2020).

[18] Dehning, J. et al. Inferring change points in the spread of COVID-19 reveals the effectiveness of interventions. Science (2020).

[19] Hethcote, H. W. The Mathematics of Infectious Diseases. SIAM Review 42, 599-653 (2000). URL https://epubs.siam.org/doi/abs/10.1137/s0036144500371907.

[20] Zhao, S. et al. Preliminary estimation of the basic reproduction number of novel coronavirus (2019-ncov) in china, from 2019 to 2020: A data-driven analysis in the early phase of the outbreak. International Journal of Infectious Diseases 92, 214 - 217 (2020). URL http://www.sciencedirect.com/science/ article/pii/S1201971220300539.

[21] Alimohamadi, Y. et al. Estimate of the Basic Reproduction Number for COVID-19: A Systematic Review and Meta-analysis. J Prev Med Public Health 53, 151-157 (2020).

[22] Liu, Y., Gayle, A. A., Wilder-Smith, A. \& Rocklöv, J. The reproductive number of COVID-19 is higher compared to SARS coronavirus. Journal of travel medicine (2020).

[23] La Salle, J. P. The stability of dynamical systems (SIAM, 1976).

[24] Wilting, J. \& Priesemann, V. Inferring collective dynamical states from widely unobserved systems. Nature Communications 9, 2325 (2018). URL https://doi.org/10.1038/s41467-018-04725-4.

[25] Li, Y. et al. The temporal association of introducing and lifting non-pharmaceutical interventions with the time-varying reproduction number (R) of SARS-CoV-2: a modelling study across 131 countries. The Lancet Infectious Diseases (2020). URL http://www.sciencedirect.com/science/article/pii/ S14/330992030/854.

[26] Holt, E. Slovakia to test all adults for SARS-CoV-2. The Lancet 396, 1386-1387 (2020). URL https: //www.thelancet.com/journals/lancet/article/PIIS0140-6736(20)32261-3/abstract.

[27] Mina, M. J., Parker, R. \& Larremore, D. B. Rethinking Covid-19 Test Sensitivity A Strategy for Containment. New England Journal of Medicine 0, null (2020). URL https://doi.org/10.1056/ NEJMP2025631.

[28] Larremore, D. B. et al. Test sensitivity is secondary to frequency and turnaround time for COVID-19 surveillance. medRxiv (2020). URL https://www.ncbi.nlm.nih.gov/pmc/articles/PMC7325181/.

[29] Kirkcaldy, R. D., King, B. A. \& Brooks, J. T. COVID-19 and Postinfection Immunity: Limited Evidence, Many Remaining Questions. JAMA 323, 2245-2246 (2020). URL https://doi.org/ 10.1001/jama.2020.7869. https://jamanetwork.com/journals/jama/articlepdf/2766097/jama_ kırkcaldy_2020_vp_20009b.pdt.

[30] Hale, T., Webster, S., Petherick, A., Phillips, T. \& Kira, B. Oxford COVID-19 Government Response Tracker, Blavatnik School of Government. . https://www.fda.gov/media/136472/download (2020).

[31] Kucharski, A. J. et al. Effectiveness of isolation, testing, contact tracing, and physical distancing on reducing transmission of SARS-CoV-2 in different settings: a mathematical modelling study. The Lancet Infectious Diseases 0 (2020).

[32] Kerr, C. C. et al. Controlling COVID-19 via test-trace-quarantine. medRxiv 2020.07.15.20154765 (2020). URL https://www.medrxiv.org/content/10.1101/2020.07.15.20154765v4.

[33] Fraser, C., Riley, S., Anderson, R. M. \& Ferguson, N. M. Factors that make an infectious disease outbreak controllable. Proceedings of the National Academy of Sciences 101, 6146-6151 (2004).

[34] Ferretti, L. et al. Quantifying SARS-CoV-2 transmission suggests epidemic control with digital contact tracing. Science $\mathbf{3 6 8}$ (2020). 
medRxiv preprint doi: https://doi.org/10.1101/2020.12.10.20247023; this version posted December 11, 2020. The copyright holder for this preprint (which was not certified by peer review) is the author/funder, who has granted medRxiv a license to display the preprint in perpetuity. All rights reserved. No reuse allowed without permission.

Low case numbers enable long-term stable pandemic control without lockdowns

[35] Lunz, D., Batt, G. \& Ruess, J. To isolate, or not to isolate: a theoretical framework for disease control via contact tracing. medRxiv 2020.05.26.20113340 (2020). URL http://medrxiv.org/content/early/ $2020 / 05 / 27 / 2020.05 \cdot 26.20113340$. abstract.

[36] Sturniolo, S., Waites, W., Colbourn, T., Manheim, D. \& Panovska-Griffiths, J. Testing, tracing and isolation in compartmental models. medRxiv (2020).

[37] Hellewell, J. et al. Feasibility of controlling COVID-19 outbreaks by isolation of cases and contacts. The Lancet Global Health (2020).

[38] Davis, E. L. et al. An imperfect tool: COVID-19'test \& trace' success relies on minimising the impact of false negatives and continuation of physical distancing. medRxiv (2020).

[39] Van Bavel, J. J. et al. Using social and behavioural science to support covid-19 pandemic response. Nature Human Behaviour 1-12 (2020).

[40] Brauner, J. M. et al. The effectiveness and perceived burden of nonpharmaceutical interventions against COVID-19 transmission: a modelling study with 41 countries. medRxiv (2020).

[41] Hsiang, S. et al. The effect of large-scale anti-contagion policies on the COVID-19 pandemic. Nature 584, 262-267 (2020). URL https://www.nature.com/articles/s41586-020-2404-8.

[42] Chu, D. K. et al. Physical distancing, face masks, and eye protection to prevent person-to-person transmission of SARS-CoV-2 and COVID-19: a systematic review and meta-analysis. The Lancet 395, 19731987 (2020). URL https://www.thelancet.com/journals/lancet/article/PIIS0140-6736(20) 31142-9/abstract.

[43] Howard, J. et al. Face Masks Against COVID-19: An Evidence Review (2020). URL https://www. preprints.org/manuscript/202004.0203/v1.

[44] Max Roser, E. O.-O., Hannah Ritchie \& Hasell, J. Coronavirus pandemic (covid-19). Our World in Data (2020). https://ourworldindata.org/coronavirus, (Europe, America, and Oceania and Asia).

[45] McDermott, J. H. \& Newman, W. G. Refusal of viral testing during the SARS-CoV-2 pandemic. Clinical Medicine (2020).

[46] Lai, C.-C. et al. Asymptomatic carrier state, acute respiratory disease, and pneumonia due to severe acute respiratory syndrome coronavirus 2 (SARS-CoV-2): facts and myths. Journal of Microbiology, Immunology and Infection (2020).

[47] Kronbichler, A. et al. Asymptomatic patients as a source of COVID-19 infections: A systematic review and meta-analysis. International Journal of Infectious Diseases (2020).

[48] Huang, L. et al. Rapid asymptomatic transmission of COVID-19 during the incubation period demonstrating strong infectivity in a cluster of youngsters aged 16-23 years outside Wuhan and characteristics of young patients with COVID-19: a prospective contact-tracing st. Journal of Infection (2020).

[49] Lavezzo, E. et al. Suppression of COVID-19 outbreak in the municipality of Vo, Italy. Nature (2020).

[50] Byambasuren, O. et al. Estimating the extent of true asymptomatic COVID-19 and its potential for community transmission: systematic review and meta-analysis. Available at SSRN 3586675 (2020).

[51] Chau, N. V. V. et al. The natural history and transmission potential of asymptomatic SARS-CoV-2 infection. Clinical Infectious Diseases (2020).

[52] Cevik, M., Kuppalli, K., Kindrachuk, J. \& Peiris, M. Virology, transmission, and pathogenesis of SARS-CoV-2. BMJ 371 (2020). URL https://www.bmj.com/content/371/bmj.m3862.

[53] Pollán, M. et al. Prevalence of SARS-CoV-2 in Spain (ENE-COVID): a nationwide, population-based seroepidemiological study. The Lancet (2020).

[54] Firth, J. A. et al. Combining fine-scale social contact data with epidemic modelling reveals interactions between contact tracing, quarantine, testing and physical distancing for controlling \{COVID $\}-19$. medRxiv (2020).

[55] Kojaku, S., Hébert-Dufresne, L. \& Ahn, Y.-Y. The effectiveness of contact tracing in heterogeneous networks. arXiv preprint arXiv:2005.02362 (2020).

[56] an der Heiden, M. \& Hamouda, O. Schätzung der aktuellen Entwicklung der SARS-CoV-2- Epidemie in Deutschland Nowcasting. Epidemiologisches Bulletin 2020, 10-15 (2020).

[57] Lauer, S. A. et al. The incubation period of coronavirus disease 2019 (COVID-19) from publicly reported confirmed cases: estimation and application. Annals of internal medicine (2020). 
medRxiv preprint doi: https://doi.org/10.1101/2020.12.10.20247023; this version posted December 11, 2020. The copyright holder for this preprint (which was not certified by peer review) is the author/funder, who has granted medRxiv a license to display the preprint in perpetuity. All rights reserved. No reuse allowed without permission.

Low case numbers enable long-term stable pandemic control without lockdowns

[58] Shampine, L. F. \& Thompson, S. Solving ddes in matlab. Applied Numerical Mathematics 37, 441-458 (2001).

[59] Jarlebring, E. Some numerical methods to compute the eigenvalues of a time-delay system using matlab. The delay e-letter 2, 155 (2008).

[60] Trefethen, L. N. Spectral methods in MATLAB (SIAM, 2000).

[61] He, X. et al. Temporal dynamics in viral shedding and transmissibility of COVID-19. Nature Medicine $1-4$ (2020).

[62] Pan, F. et al. Time course of lung changes on chest CT during recovery from 2019 novel coronavirus (COVID-19) pneumonia. Radiology 200370 (2020).

[63] Ling, Y. et al. Persistence and clearance of viral RNA in 2019 novel coronavirus disease rehabilitation patients. Chinese medical journal (2020).

[64] Bar-On, Y. M., Flamholz, A., Phillips, R. \& Milo, R. Science forum: Sars-cov-2 (covid-19) by the numbers. Elife 9, e57309 (2020).

[65] Siegenfeld, A. F. \& Bar-Yam, Y. The impact of travel and timing in eliminating covid-19. Communications Physics 3, 204 (2020). URL https://doi.org/10.1038/s42005-020-00470-7.

[66] Bittihn, P. \& Golestanian, R. Containment strategy for an epidemic based on fluctuations in the SIR model. arXiv:2003.08784 [physics, q-bio] (2020). URL http://arxiv.org/abs/2003.08784. ArXiv: 2003.08784 .

[67] Levin, A. T. et al. Assessing the age specificity of infection fatality rates for COVID-19: Systematic review, meta-analysis, and public policy implications. medRxiv (2020). URL https://www.medrxiv. org/content/early/2020/09/26/2020.07.23.20160895. MedRxiv: 10.1101/2020.07.23.20160895v5, https://www.medrxiv.org/content/early/2020/09/26/2020.07.23.20160895.full.pdf.

[68] Great Barrington declaration and petition, https://gbdeclaration.org/ (2020).

[69] Bhopal, R. S. COVID-19 zugzwang: Potential public health moves towards population (herd) immunity. Public Health in Practice 1, 100031 (2020). URL http://www.sciencedirect.com/science/article/ pii/S2666535220300306.

[70] Salvatier, J., Wiecki, T. V. \& Fonnesbeck, C. Probabilistic programming in Python using PyMC3. PeerJ Computer Science 2, e55 (2016). URL https://peerj.com/articles/cs-55.

\section{Author Contributions}

S.C, J.D., and V.P. designed the research. S.C., J.D., S.M., and P.S. conducted the research. All authors analysed the data. S.C., S.M., and P.S. created the figures. All authors wrote the paper.

\section{Competing Interests}

The authors declare no competing interests.

\section{Data availability}

Both data ${ }^{44}$ and analysis code https://github.com/Priesemann-Group/covid19_research/tree/ master/circuit breaker are available online. Additionally, an interactive platform to simulate scenarios different from those presented here will be soon available (beta-version). The permanent link will be provided in the same GitHub repository.

\section{Acknowledgements}

We thank Melanie Brinkmann and Álvaro Olivera-Nappa for their helpful comments and encouraging feedback. We thank M. Loidolt, Michael Wibral and Johannes Zierenberg from our group for carefully reading, commenting and improving the manuscript. We thank the Priesemann group for exciting discussions and for their valuable input. Funding: All authors received support from the Max-Planck-Society. JD and PS acknowledge funding by SMARTSTART, the joint training program in computational neuroscience by the VolkswagenStiftung and the Bernstein Network. 


\section{S1 Methods}

Model overview. We model the spreading dynamics of SARS-CoV-2 as the sum of contributions from two pools of infectious individuals, i.e. quarantined-isolated $I^{Q}$ and hidden non-isolated $I^{H}$ individuals, while also modelling the infectivity timeline through the incorporation of compartments for individuals exposed to the virus $\left(E^{Q}, E^{H}\right)$, following an SEIR-like formalism. The quarantined infectious pool $\left(I^{Q}\right)$ contains cases revealed through testing or by contact tracing and subsequently sent to quarantine-isolation to avoid further contacts as well as possible. In contrast, in the hidden infectious pool $\left(I^{H}\right)$, infections spread silently and only become detectable when individuals develop symptoms and get tested, via random testing in the population or as part of the chain of contacts of recently identified individuals. This second pool $\left(I^{H}\right)$ is called the hidden pool; individuals in this pool are assumed to exhibit the general population's behaviour, thus of everyone who is not aware of being infected. Healthy individuals that can be infected belong to the susceptible pool $S$, while we assume that, after they recover and for the relatively short time-frame here studied, they remain immunised in the $R$ compartment. For a graphical representation of the model, the reader is referred to Supplementary Fig. 52. We model the mean-field interactions between compartments by transition rates, determining the timescales involved. These transition rates can implicitly incorporate both the time course of the disease and the delays inherent to the TTI process. Individuals exposed to the virus become infectious after the latent period, modelled by the We distinguish between symptomatic and asymptomatic carriers - this is central when exploring different testing strategies (as detailed below). We also include the effects of non-compliance, modelled as a higher asymptomatic ratio, and imperfect contact tracing, including an explicit delay between testing and tracing of contacts. In the different scenarios analysed, we include a nonzero influx $\Phi_{t}$ of new cases that acquired the virus from outside. Even though this influx makes a complete eradication of SARS-CoV-2 impossible, different outcomes in the spreading dynamics might arise depending on both contact intensity and TTI. We then investigate the system's stability and dynamics, aiming to control the spread with a low total number of cases without necessitating a too large reduction of infectious contacts.

\section{S1.1 Spreading Dynamics}

Concretely, we use a modified SEIR-type model, where infected individuals can be either symptomatic or asymptomatic. They belong to hidden $\left(E^{H}, I^{H}\right)$ or a quarantined $\left(E^{Q}, I^{Q}\right)$ pools of infections, thus creating in total one compartment of susceptible $(S)$, two compartments of exposed individuals $\left(E^{H}, E^{Q}\right)$, four compartments of infectious individuals $\left(I^{H, s}, I^{H, a}, I^{Q, s}, I^{Q, a}\right)$, and one compartment for recovered/removed individuals $(R)$.

New infections are asymptomatic with a ratio $\xi$, the others are symptomatic. In all compartments individuals are removed with a rate $\gamma$ because of recovery or death (Supplementary Table \$1 for all parameters).

In the hidden pools, the disease spreads according to the contact patterns of the population, which can be expressed as a fraction $k_{t}$ of the intensity they had before COVID-19 related contact-restrictions. Defining $R_{0}$ as the base reproduction number without contact restrictions, the reproduction number of the hidden pool $I^{H}$ is given by $\left(1-k_{t}\right) R_{0}$. This reproduction number reflects the disease spread in the general population, without testing induced isolation of individuals. Additionally, the hidden pool receives a mobility-induced influx $\Phi_{t}$ of new infections. Cases are removed from the hidden pool (i) when detected by TTI and put into the quarantined pool $I^{Q}$, or (ii) due to recovery or death.

The quarantined exposed and infectious pools $\left(E^{Q}, I^{Q}\right)$ contain those infected individuals who have been tested positive as well as their positively tested contacts. Infectious individuals in $I^{Q}$ are (imperfectly) isolated, we assume their contacts have been reduced to a fraction $(\nu+\epsilon)$ of the ones they had in preCOVID-19 times, of which only $\nu$ are captured by the tracing efforts of the health authorities, and the subsequent infections remain quarantined, thus entering the $E^{Q}$ pool and afterwards the $I^{Q}$ pool. The remaining fraction of produced infections, $\epsilon$, are missed and act as an influx to the hidden pools $\left(E^{H}\right)$. Therefore, the overall reproduction number in the $I^{Q}$ pool is $(\nu+\epsilon) R_{0}$.

As our model is an expanded SEIR-model, it assumes post-infection immunity, which is a realistic assumption given the limited time-frame considered in our analysis. Our model can also reflect innate immunity; one then has to rescale the population or the reproduction number. The qualitative behaviour of the dynamics is not expected to change. 
Low case numbers enable long-term stable pandemic control without lockdowns

\section{S1.2 Parameter Choices and Scenarios}

For any testing strategy, the fraction of infections that do not develop any symptoms across the whole infection timeline is an important parameter, and this also holds for testing strategies applied to the case of SARS-CoV-2. In our model this parameter is called $\xi^{\text {ap }}$ and includes beside true asymptomatic infections $\xi$ also the effect of individuals that avoid testing ${ }^{4.5}$. The exact value of the fraction of asymptomatic infections $\xi$, however, is still fraught with uncertainty, and it also depends on age ${ }^{46 ; 477 ; 48}$. While early estimates were as high as $50 \%$ (for example ranging from $26 \%$ to $63 \%{ }^{49}$ ), these early estimates suffered from reporting bias, small sample sizes and sometimes included pre-symptomatic cases as well ${ }^{\text {III }}$. Recent studies estimate the asymptomatic transmission to be more minor ${ }^{[52}$, estimates of the fraction of asymptomatic carriers range between $12 \%$ and $33 \%$.

Another crucial parameter for any TTI strategy is the reproduction number of the hidden infections. This parameter is by definition impossible to measure, but it is typically the main driver of the spreading dynamics. It depends mainly on the contact behaviour of the population, and ranges from $R_{0}$ in the absence of contact restrictions to values below 1 during strict lockdown ${ }^{\square 1}$. Herein we decided to include instead the reduction of contacts with respect to the pre-COVID-19 base-line $k_{t}$ to represent the reproduction number of hidden infections $\left(1-k_{t}\right) R_{0}$. For the default parameters of our model, we evaluated different levels of contact reduction $k_{t}$.

\section{S1.3 Testing-and-Tracing strategies}

We consider a testing-and-tracing strategy: symptom-driven testing and specific testing of traced contacts, with a subsequent of the cases tested positive. Our model can also include random testing, but this case is only explored in Figure 4 of this paper.

Symptom-driven testing is defined as applying tests to individuals presenting symptoms of COVID-19. In this context, it is important to note that non-infected individuals can have symptoms similar to those of COVID-19, as many symptoms are rather unspecific. Although symptom-driven testing suffers less from imperfect specificity, it can only uncover symptomatic cases that are willing to be tested (see below). Here, symptomatic, infectious individuals are transferred from the hidden to the traced pool at rate $\lambda_{s}$.

We define $\lambda_{s}$ as the daily rate at which symptomatic individuals get tested, among the subset who are willing to get tested, because of surveillance programs or self-report. As default value we use $\lambda_{s}=0.25$, which means that, on average, an individual willing to get tested that develops COVID-19-specific symptoms would get a test within 4 days from the end of the latency period. Testing and isolation happens immediately in this model, but their report into the observed new daily cases $\hat{N}^{\text {obs }}$ is delayed, and so is the tracing of their contacts.

Tracing contacts of positively tested infectious individuals presents a very specific test strategy, and is expected to be effective in breaking the infection chains, if contacts self-isolate sufficiently quickly However as every implementation of a TTI strategy is bound to be imperfect, we assume that only a fraction $\eta<1$ of all contacts can be traced. These contacts, if tested positive, are then transferred from the hidden to the quarantined infectious pools $\left(I^{H} \rightarrow I^{Q}\right)$ with an average delay of $\tau=2$ days. The parameter $\eta$ effectively represents the fraction of secondary and tertiary infections that are found through contact-tracing. As this fraction decreases when the delay between testing and contact-tracing increases we assumed a default value of $\eta=0.66$, i.e. on average only two thirds of subsequent offspring infections are prevented. Contact tracing is mainly done by the health authorities in Germany, and this clearly limits the maximum number $N_{\max }^{\text {test }}$ of new cases observed through testing $N^{\text {test }}$, for which contact tracing is still functional.

Random testing is defined here as applying tests to individuals irrespective of their symptom status, or whether they belonging to the contact-chain of other infected individuals. In our model, random testing transfers infected individuals from the hidden to the quarantined infectious pools with fixed rate $\lambda_{r}$, irrespective of them showing symptoms or not. In reality, random testing is often implemented as situation-based testing for a sub-group of the population, e.g. at a hot-spot, for groups at risk, or for people returning from travel. Such situation-based strategies would be more efficient than the random testing assumed in this model, which may be unfeasible in a country level due to testing limitations ${ }^{13}$.

\section{S1.4 Lockdown modelling}

To assess the effectiveness of lockdowns in the broad spectrum of contact-ban governmental interventions, we model how the reduction of contacts and the duration of such restrictive regimes help lower case numbers. 
Low case numbers enable long-term stable pandemic control without lockdowns

We model contact reductions as reductions in the reproduction number of the hidden population, which for these matters is presented as percentages of the basic reproduction number $R_{0}$, which sets the pre-COVID-19 base-line for the number of close contacts.

For the sake of simplicity, we assume the lockdown scenarios have three stages: i) an uncontrolled regime, where the TTI capacity is overwhelmed because of high case numbers and unsustainable contact intensity, reflected by a high value of $k_{t}=k_{0}$ and high influx of infections $\Phi_{t}$. ii) Strong reduction of contacts $k_{0} \rightarrow k_{\mathrm{LD}}$ and borders closing $\Phi_{t} \rightarrow \Phi_{\mathrm{LD}}$. iii) Relaxation of measures to a new level, where the system should be again stable, within the TTI-based stability $k_{\mathrm{LD}} \rightarrow k_{\mathrm{nLD}}$, and borders would be open $\Phi_{\mathrm{LD}} \rightarrow \Phi_{\mathrm{nLD}}$. All the changes between the different regimes $\mathrm{i} \rightarrow \mathrm{i} \rightarrow \mathrm{iii}$ are modelled as linear ramps for both parameters, which take $D_{\text {ramp }}=7$ days to reach their set-point. The duration of the lockdown $D_{\mathrm{L}}$, namely, the time-frame between the start of the restrictive measures and the beginning of their relaxation, is measured in weeks, and its default length - for analysis purposes - is four weeks.

\section{S1.5 Model Equations}

The contributions of the spreading dynamics and the TTI strategies are summarised in the equations below. They govern the dynamics of case numbers between the different susceptible-exposed-infectious-recovered (SEIR) pools, both hidden (non-isolated) and quarantined. We assume a regime where most of the population is susceptible, and the time-frame analysed is short enough to assume post-infection immunity. Thus, the dynamics are completely determined by the spread (characterised by the reproduction numbers $\left(1-k_{t}\right) R_{0}$ and $(\nu+\epsilon) R_{0}$ ), transition from exposed to infectious (at rate $\rho$ ), recovery (characterised by the recovery rate $\gamma$ ), external influx $\Phi_{t}$ and the impact of the TTI strategies:

$$
\begin{aligned}
& \frac{d S}{d t} \quad=-\underbrace{\gamma\left(1-k_{t}\right) R_{0} \frac{S}{M} I^{H}}_{\text {hidden contagion }} \quad-\underbrace{\gamma(\nu+\epsilon) R_{0} \frac{S}{M} I^{Q}}_{\text {traced contagion }}-\underbrace{\frac{S}{M} \Phi_{t}}_{\text {ext. influx }}, \\
& \frac{d E^{Q}}{d t} \quad=\underbrace{\gamma \nu R_{0} \frac{S}{M} I^{Q}}_{\text {traced contagion }} \\
& \frac{d E^{H}}{d t}=\underbrace{\gamma \frac{S}{M}\left(\left(1-k_{t}\right) R_{0} I^{H}+\epsilon R_{0} I^{Q}\right)}_{\text {hidden contagion }}-\underbrace{\chi_{\tau} N^{\text {traced }}}_{\text {contact tracing }}-\underbrace{\rho E^{H}}_{\text {end of latency }}, \\
& \frac{d I^{Q}}{d t} \quad=\underbrace{\rho E^{Q}-\gamma I^{Q}}_{\text {spreading dynamics }}+\underbrace{N^{\text {test }}}_{\text {testing }} \\
& \frac{d I^{H}}{d t} \quad=\underbrace{\rho E^{H}-\gamma I^{H}}_{\text {spreading dynamics }}-\underbrace{N^{\text {test }}}_{\text {testing }} \\
& \frac{d I^{H, s}}{d t}=\underbrace{(1-\xi) \rho E^{H}-\gamma I^{H, s}}_{\text {spreading dynamics }}-\underbrace{N_{s}^{\text {test }}}_{\text {testing }} \\
& I^{H, a}=I^{H}-I^{H, s} \text {, } \\
& \frac{d R}{d t} \quad=\underbrace{\gamma\left(I^{Q}+I^{H}\right)}_{\text {recovered/removed individuals }} .
\end{aligned}
$$

\section{S1.6 Initial conditions}

Let $x$ be the vector collecting the variables of all different pools:

$$
x=\left[S, E^{Q}, E^{H}, I^{Q}, I^{H}, I^{H, s}, R\right] .
$$


We assume a population size of $M=10^{6}$ individuals, so that $\sum_{i \neq 6} x_{i}=M$, and a prevalence of $I_{0}=200$ infections per million, so that $I^{Q}(0)=I_{0}$. Assuming that the hidden amount of infections is in the same order of magnitude $I_{0}$, we would have $I^{H}(0)=I_{0}, I^{H, s}(0)=(1-\xi) I_{0}$. We would expect the exposed individuals to scale with $\left(1-k_{t}\right) R_{0} I_{0}$, but we rather assume them to have the same size of the corresponding infectious pool. To calculate the initially susceptible individuals, we use $S(0)=1-\sum_{i \neq\{1,6\}} x_{i}$.

\section{S1.7 Effect of delays and capacity limit on the effectiveness of TTI strategies}

In this section we discuss further details on the derivation of the different parameters and variables involved in equations (四)-(छ). First, as we assume contact tracing to be effective after a delay of $\tau$ days, some of the individuals who acquired the infection from those recently tested might have also become infectious by the time of tracing. Moreover, a fraction of those who became infectious might also have been tested by the tracing time, should they have developed symptoms.

Furthermore, we give explicit forms for $N^{\text {test }}$ and $N^{\text {traced }}$ the number of cases identified respectively by testing and contact-tracing. When surpassing TTI capacity we assume that both testing and contact-tracing change their dynamics simultaneously. This happens when the daily amount of cases identified by testing $N^{\text {test }}$ overpasses the TTI threshold $N_{\max }^{\text {test }}$. After being overwhelmed, the overhead testing would change its rate $\lambda_{s} \rightarrow \lambda_{s}^{\prime}$, as only patients with a more specific set of symptoms would be tested. Nonetheless, the contact-tracing efforts can only follow the contacts of those $N_{\max }^{\text {test }}$ observed cases, identifying a fraction $\eta$ of the offspring infections they produced in their infectious period spent in unawareness of their state. The case of random testing is analysed in the Supplementary Section 1.

\section{S1.7.1 Limited testing capacity leading to lower testing rates}

In the first stages of an outbreak, individuals with any symptoms from the broad spectrum of COVID-19related symptoms would be tested, disregarding how specific those symptoms are. At this stage, we assume that the rate at which symptomatic individuals are tested is $\lambda_{s}$, such that the number of individuals identified through testing (which, for simplicity is assumed to be solely symptom-driven) is given by

$$
N_{s}^{\text {test }}=\lambda_{s} I^{H, s}
$$

When reaching the daily number $N_{\max }^{\text {test }}$ of positive tests the testing capacity is reached. We then assume that further tests are only carried out for a more specific set of symptoms, leading to a smaller fraction of the population being tested. We therefore implement the testing capacity as a soft threshold. Assuming that after reaching $N_{\max }^{\text {test }}$, the testing rate for further cases would decrease to $\lambda_{s}^{\prime}$, the testing term $N^{\text {test }}$ would be given by

$$
N_{s}^{\mathrm{test}}=\lambda_{s} \min \left(I^{H, s}, I_{\max }^{H, s}\right)+\lambda_{s}^{\prime} \max \left(0, I^{H, s}-I_{\max }^{H, s}\right)
$$

where $I_{\max }^{H, s}$ represent the size of the infectious -symptomatic, hidden- pool i.e, $I_{\max }^{H, s}=\frac{N_{\max }^{\text {test }}}{\lambda_{s}}$.

\section{S1.7.2 Modelling the number of traced individuals}

To calculate the number of traced individuals, we assume that a fraction $\eta$ of the newly tested individuals' contacts, and therefore their offspring infections, will be traced and subsequently quarantined. However these tested individuals stay on average a shorter amount of time in the infectious pool than the untested ones because they are quarantined, thus removed earlier from the pool. The number of offspring infections has therefore to be corrected by a factor, the average residence time in the infectious pool. For the case $\lambda_{r}=0$, the average residence time is $\frac{1}{\gamma+\lambda_{s}}$, whereas the average residence time of untested individuals is $\frac{1}{\gamma}$. Dividing these two times gives us the wanted correction factor. The number of traced persons $N^{\text {traced }}$ at time $t$ is therefore:

$$
N^{\text {traced }}(t)=\eta R_{t-\tau}^{H} \frac{\gamma}{\gamma+\lambda_{s}} N^{\text {test }}(t-\tau) .
$$


Otherwise, when the TTI capacity is overwhelmed, we assume that the number of traced individuals is limited, that only the contacts of $N_{\max }^{\text {test }}$ individuals (already introduced in the previous section) can be traced:

$$
N^{\text {traced }}(t)=\eta R_{t-\tau}^{H} \frac{\gamma}{\gamma+\lambda_{s}} N_{\max }^{\text {test }}
$$

\section{S1.7.3 Individuals becoming infectious or being tested by the time of tracing}

The traced individuals are removed from either the exposed hidden pool $E^{H}$ or from the infectious hidden pool $I^{H}$ after a delay of $\tau$ days after testing. As we assume a tracing delay $\tau$ of only 2 days, a majority of the traced individuals are still be in exposed compartment. However, some might already become infectious by that time. To calculate the exact fraction of individuals remaining in the hidden exposed pool by the time of tracing, we proceed as follows. Let $s \in I_{\tau}=[0, \tau]$ be the time elapsed from the moment of testing. The emptying of the normalised exposed compartment (denoted $\widetilde{E^{H}}$ ) due to progression to the infectious stage follows first-order kinetics:

$$
\frac{d \widetilde{E^{H}}}{d s}=-\widetilde{E^{H}}, \quad \widetilde{E^{H}}(0)=1
$$

The solution of (피) is given by $\widetilde{E^{H}(s)}=\exp (-\rho s)$. Therefore, we define $\chi_{\tau}$ as the fraction of the traced individuals remaining in the $E^{H}$ compartment at $s=\tau$ :

$$
\chi_{\tau}=\exp (-\rho \tau)
$$

The remaining individuals are removed from the infectious compartment, which are then simply described by the fraction

$$
\chi_{r}=1-\chi_{\tau}
$$

This however only holds for the asymptomatic hidden infectious pool. For the symptomatic hidden pool $I^{H, s}$, we do not want to remove the individuals which have already been tested, as they would be removed twice. For modelling the fraction of non-tested individuals remaining in the normalised symptomatic infectious compartment (denoted $\widetilde{I^{H, s}}$ ), we couple two first-order kinetics:

$$
\frac{d \widetilde{I^{H, s}}}{d s}=\lambda_{s} \widetilde{I^{H, s}}+\widetilde{E^{H}}, \quad \widetilde{I^{H, s}}(0)=0
$$

The solution of (마) depends on whether $\lambda_{s}=\rho$ or not. The solution at $s=\tau$ which is the fraction of traced individuals removed from $I^{H, s}$ is given by:

$$
\chi_{s, r}= \begin{cases}\rho \tau \exp (-\rho \tau) & \text { if } \lambda_{s} \approx \rho \\ \frac{\rho}{\lambda_{s}-\rho}\left(\exp (-\rho \tau)-\exp \left(-\lambda_{s} \tau\right)\right) & \text { else. }\end{cases}
$$

For the case $\lambda_{r} \neq 0$, the reader is referred to the Supplementary Note 4.

\section{S1.8 Central epidemiological parameters that can be observed}

In the real world, the disease spread can only be observed through testing and tracing. While the true number of daily infections $N$ is a sum of all new infections in the hidden and traced pools, the observed number of daily infections $\hat{N}^{\text {obs }}$ is the number of new infections discovered by testing, tracing, and surveillance of the contacts of those individuals in the quarantined infectious pool $I^{Q}$, delayed by a variable reporting time. This includes internal contributions and contributions from testing and tracing: 


$$
\begin{aligned}
N & =\underbrace{\gamma\left(1-k_{t}\right) R_{0} \frac{S}{M} I^{H}}_{\text {hidden contagion }}+\underbrace{\gamma(\nu+\epsilon) R_{0} \frac{S}{M} I^{Q}}_{\text {traced contagion }}+\underbrace{\frac{S}{M} \Phi_{t}}_{\text {ext. influx }} \\
\hat{N}^{\text {obs }} & =[\underbrace{\rho E^{Q}}_{\text {traced contagion }}+\underbrace{N^{\text {test }}+\left(\chi_{s, r}(1-\xi)+\chi_{r} \xi\right) N^{\text {traced }}}_{\text {TTI }}] \circledast \mathcal{K},
\end{aligned}
$$

where $\circledast$ denotes a convolution and $\mathcal{K}$ an empirical probability mass function that models a variable reporting delay, inferred from German data (as the RKI reports the date the test is performed, the delay until the appearance in the database can be inferred): The total delay between testing and reporting a test corresponds to one day more than the expected time the laboratory takes for obtaining results, which is defined as follows: from testing, $50 \%$ of the samples would be reported the next day, $30 \%$ the second day, $10 \%$ the third day, and further delays complete the remaining $10 \%$, which for simplicity we will truncate at day four. Considering the extra day needed for reporting, the probability mass function for days 0 to 5 would be given by $\mathcal{K}=[0,0,0.5,0.3,0.1,0.1]$. The spreading dynamics are usually characterized by the observed reproduction number $\hat{R}_{t}^{\text {obs }}$, which is calculated from the observed number of new cases $\hat{N}^{\text {obs }}(t)$. We here use the definition underlying the estimates that are published by Robert-Koch-Institute, the official body responsible for epidemiological control in Germany daily new cases $N$ separated by 4 days (the assumed serial interval of COVID-19 ${ }^{[57}$ ):

$$
\hat{R}_{t}^{\mathrm{obs}}=\frac{\hat{N}^{\mathrm{obs}}(t)}{\hat{N}^{\mathrm{obs}}(t-4)}
$$

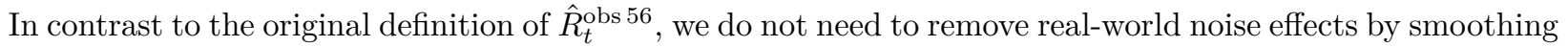
this ratio.

\section{S1.9 Numerical calculation of solutions and critical values.}

The numerical solution of the delay differential equations governing our model were obtained using a versatile solver that tracks discontinuities and integrates with the explicit Runge-Kutta (2,3) pair, @dde23 implemented in MATLAB (version 2020a), with default settings. This algorithm allows the solution of nonstiff systems of differential equations in the shape $y^{\prime}(t)=f\left(t, y(t), y\left(t-\tau_{1}\right), \ldots, y\left(t-\tau_{k}\right)\right.$, for a set of discrete lags $\left\{\tau_{i}\right\}_{i=1}^{k}$. Suitability and details on the algorithm are further discussed in ${ }^{20 \mathrm{x}}$.

To derive the tipping point between controlled and uncontrolled outbreaks (e.g. critical, minimal required contact reduction $k_{t}^{\text {crit }}$ for both stability and metastability), and to plot the stability diagrams, we used the @fzero MATLAB function, and the linear approximation of the system of DDE (区) $-\left(\right.$ (G) for the $\frac{S}{M} \approx 1$ limit. This function uses a combination of bisection, secant, and inverse quadratic interpolation methods to find the roots of a function. For instance, following the discussion of Supplementary Section 1, The different critical values for the contact reduction $k_{t}^{\text {crit }}$ were determined by systematically solving the nonlinear eigenvalues problem for stability ${ }^{\text {, }}$, where the solution operation was approximated with a Chebyshev differentiation matrix 
medRxiv preprint doi: https://doi.org/10.1101/2020.12.10.20247023; this version posted December 11, 2020. The copyright holder for this preprint (which was not certified by peer review) is the author/funder, who has granted medRxiv a license to display the preprint in perpetuity. All rights reserved. No reuse allowed without permission.

Low case numbers enable long-term stable pandemic control without lockdowns

Supplementary Table S1: Model parameters.

\begin{tabular}{|c|c|c|c|c|c|}
\hline Parameter & Meaning & $\begin{array}{l}\text { Value } \\
\text { (default) }\end{array}$ & Range & Units & Source \\
\hline$M$ & Population size & 1000000 & & people & Assumed \\
\hline$R_{0}$ & Basic reproduction number & 3.3 & $2.2-4.4$ & - & $201 ; 21 ; ; 22$ \\
\hline$\nu$ & Registered contacts (quarantined) & 0.075 & & - & Assumed \\
\hline$\epsilon$ & Lost contacts (quarantined) & 0.05 & & - & Assumed \\
\hline$\gamma$ & Recovery/removal rate & 0.10 & $0.08-0.12$ & day $^{-1}$ & [1];62;;63 \\
\hline$\xi$ & Asymptomatic ratio & 0.32 & $0.15-0.43$ & - & 国;35;49 \\
\hline$\lambda_{s}$ & Symptom-driven testing rate & 0.25 & $0-1$ & day $^{-1}$ & Assumed \\
\hline$\lambda_{s}^{\prime}$ & $\begin{array}{l}\text { Symptom-driven testing rate (reduced } \\
\text { capacity) }\end{array}$ & 0.1 & & day $^{-1}$ & Assumed \\
\hline$\eta$ & Tracing efficiency & 0.66 & & - & Assumed \\
\hline$\tau$ & Contact tracing delay & 2 & & days & Assumed \\
\hline$N_{\max }^{\text {test }}$ & Maximal tracing capacity & 50 & $10-75$ & cases day $^{-1}$ & Assumed \\
\hline$\Phi_{t}$ & External influx & & & cases day $^{-1}$ & Assumed \\
\hline$\rho$ & Exposed-to-infectious rate & 0.25 & & day $^{-1}$ & 64;[15] \\
\hline$D_{\mathrm{L}}$ & lockdown duration & 4 & $0-8$ & weeks & Assumed \\
\hline$D_{\text {ramp }}$ & Phase-transition duration (lockdown) & 1 & & weeks & Assumed \\
\hline & Baseline contact reduction & $20 \%$ & & - & Assumed \\
\hline$k_{\mathrm{LD}}$ & Contact reduction during lockdown & $75 \%$ & & - & Assumed \\
\hline$k_{\mathrm{nLD}}$ & Contact reduction post lockdown & $40 \%$ & & - & Assumed \\
\hline$\Phi_{\mathrm{LD}}$ & Ext. influx (during lockdown) & 0.1 & & cases day $^{-1}$ & Assumed \\
\hline$\Phi_{\mathrm{nLD}}$ & Ext. influx (post lockdown) & 1 & & cases day $^{-1}$ & Assumed \\
\hline$\chi_{\tau}$ & $\begin{array}{l}\text { Fraction of contacts traced before be- } \\
\text { coming infectious }\end{array}$ & 0.61 & & - & eq (며) \\
\hline$\chi_{s, r}$ & $\begin{array}{l}\text { Fraction of contacts traced after be- } \\
\text { coming infectious, before being tested } \\
\text { (symptomatic and random) }\end{array}$ & 0.30 & & - & eq (एष) \\
\hline$\chi_{r}$ & $\begin{array}{l}\text { Fraction of contacts traced after becom- } \\
\text { ing infectious, before being tested (ran- } \\
\text { dom) }\end{array}$ & 0.39 & & - & eq (마) \\
\hline
\end{tabular}

\section{S2 Supplementary Notes}

\section{S2.1 Strategies to face COVID-19 differ among countries.}

Very low case numbers and even local eradication has been achieved by several South- and East-Asian countries, as well as Australia and New-Zealand. By aiming for local eradication, these countries reached very low values below one daily new case per million (median 0.5 , Fig. \$3. C). If local eradication is successful, these countries can profit from the absorbing state of zero SARS-CoV-2 infections, i.e. after local eradication new infection chains are only started if a virus is de novo carried into the country ${ }^{65 ; 66}$. However, the local eradication is constantly put at risk by undetected influx of new viruses from abroad, requiring very strict quarantine for international travel, and - once the spread got out of control - decisive action to completely stop all infection chains. However, the more countries adhere to this strategy successfully, the closer one may get to global eradication.

In many European countries over the summer, case numbers were relatively low, typically around 10 daily new cases per million (Fig. 5.3 B). During that time, contacts were in general only mildly or moderately restricted, and containment was complemented by hygiene, masks, and other preventive measures. However, in summer and autumn, most European countries developed a second wave Fig. ??. The causes are certainly diverse, from increasing contact rates to seasonal effects and travel-related influx. Seasonal effects alone cannot explain the second wave, as neighbouring countries like Portugal versus Spain, or Finland versus 
Low case numbers enable long-term stable pandemic control without lockdowns

Supplementary Table S2: Model variables.

\begin{tabular}{|c|c|c|c|}
\hline Variable & Meaning & Units & Explanation \\
\hline$S$ & Susceptible pool & people & non-infected people that may acquire the virus. \\
\hline$E^{Q}$ & $\begin{array}{l}\text { Exposed pool (quaran- } \\
\text { tined) }\end{array}$ & people & Total quarantined exposed people. \\
\hline$E^{H}$ & Exposed pool (hidden) & people & Total non-traced, non-quarantined exposed people. \\
\hline$I^{H, s}$ & $\begin{array}{l}\text { Infectious pool (hidden, } \\
\text { symptomatic) }\end{array}$ & people & Non-traced, non-quarantined people who are symptomatic. \\
\hline$I^{H}$ & Infectious pool (hidden) & people & Total non-traced, non-quarantined infectious people. \\
\hline$I^{Q}$ & $\begin{array}{l}\text { Infectious pool (quaran- } \\
\text { tined) }\end{array}$ & people & Total quarantined infectious people. \\
\hline$N$ & New infections (Total) & cases day $^{-1}$ & Given by: $N=\gamma\left(1-k_{t}\right) R_{0} I^{H}+\gamma(\nu+\epsilon) R_{0} I^{Q}+\frac{S}{M} \Phi_{t}$ \\
\hline$k_{t}$ & Contact reduction & $\%$ & $\begin{array}{l}\text { Reduction of infectious contacts, related to pre-COVID- } 19 \\
\text { times. }\end{array}$ \\
\hline$\hat{N}^{\text {obs }}$ & $\begin{array}{l}\text { Observed new infections } \\
\text { (influx to traced pool) }\end{array}$ & cases day $^{-1}$ & $\begin{array}{l}\text { Daily new cases, observed from the quarantined pool; de- } \\
\text { layed because of imperfect reporting and realistic contact } \\
\text { tracing. }\end{array}$ \\
\hline$\hat{R}_{t}^{\mathrm{obs}}$ & $\begin{array}{l}\text { Observed } \\
\text { number }\end{array}$ & - & $\begin{array}{l}\text { The reproduction number that can be estimated only from } \\
\text { the observed cases: } \hat{R}_{t}^{\text {obs }}=\hat{N}^{\mathrm{obs}}(t) / \hat{N}^{\mathrm{obs}}(t-4) \text {. }\end{array}$ \\
\hline
\end{tabular}
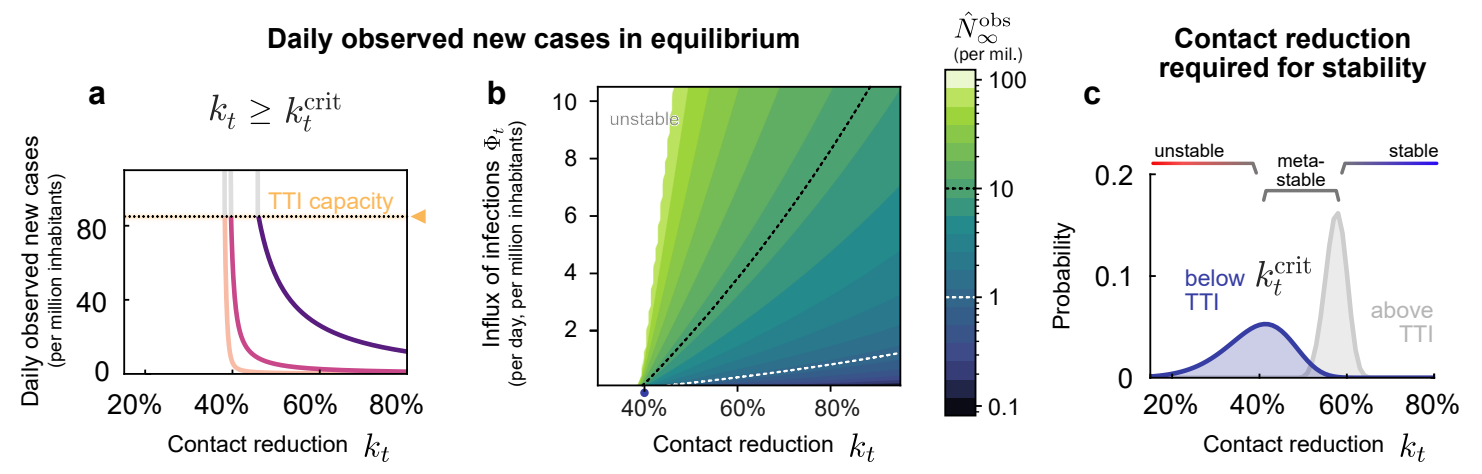

Supplementary Figure S1: In the stable and metastable regimes, daily new cases approach an equilibrium value $\hat{N}_{\infty}^{\text {obs }}$ that depends on contact reduction $k_{t}$ and external influx of new cases $\Phi_{t}$. a: The equilibrium value $\hat{N}_{\infty}^{\text {obs }}$ increases steeply with lower $k_{t}$ and then destabilises when surpassing a critical value $k_{t}^{\text {crit }}$. Thus, contact reduction has to be sufficiently strong to keep the case numbers within TTI capacity. A certain degree of external influx $\Phi_{t}$ can be compensated, but in general, $\Phi_{t}$ can put stability at risk. $\Phi_{t}=1$ one daily new case per million is our default parameter for the influx. b: The $\hat{N}_{\infty}^{\text {obs }}$ is below 10 for a large fraction of parameter combinations, $k_{t}$ and $\Phi_{t}$, thus well below the capacity limit of TTI. c: The critical value $k_{t}^{\text {crit }}$, which marks the transition between stable and (meta)stable spread; thus $k_{t}^{\text {crit }}$ displays the minimal contact reduction that is required to control the spread (and stabilise case numbers). If case numbers are below the TTI capacity limit, a contact reduction of at least $k_{t}^{\text {crit }}=39 \%$ is required for stabilisation (blue). If case numbers, however, are above the TTI limit, stronger contact reduction is necessary for stabilisation $\left(k_{t}^{\text {crit }}=58 \%\right.$, grey). Confidence intervals originate from error propagation of the uncertainty of the underlying model parameters. All model parameters are listed in Supplementary Table $\mathbf{5} 3$ and the full uncertainty analysis is in Fig. 54].

Sweden show greatly different dynamics $\left(\right.$ see $\left.^{\sqrt{44}}\right)$. Hence, it seems to be possible to maintain an equilibrium at fairly low case numbers, but that equilibrium is fragile at high case numbers and novel waves can emerge at any time.

Sustained high levels of case numbers have been observed in several countries such that TTI probably couldn't be performed effectively. Around 130 daily new cases per million have been observed e.g. in many (but not all) American countries (median 129.54, Fig. 5.3 A). It shows that high levels of daily new infections can be maintained in principle. However the stringency of interventions is similar or higher compared to other countries (see ${ }^{\text {(3II)}}$ ), and even with these high numbers it will probably take about $200.000 / 150=1333$ to $700.000 / 150=4666$ days, thus several years, until 20 to $70 \%$ of the population is infected and herd immunity 


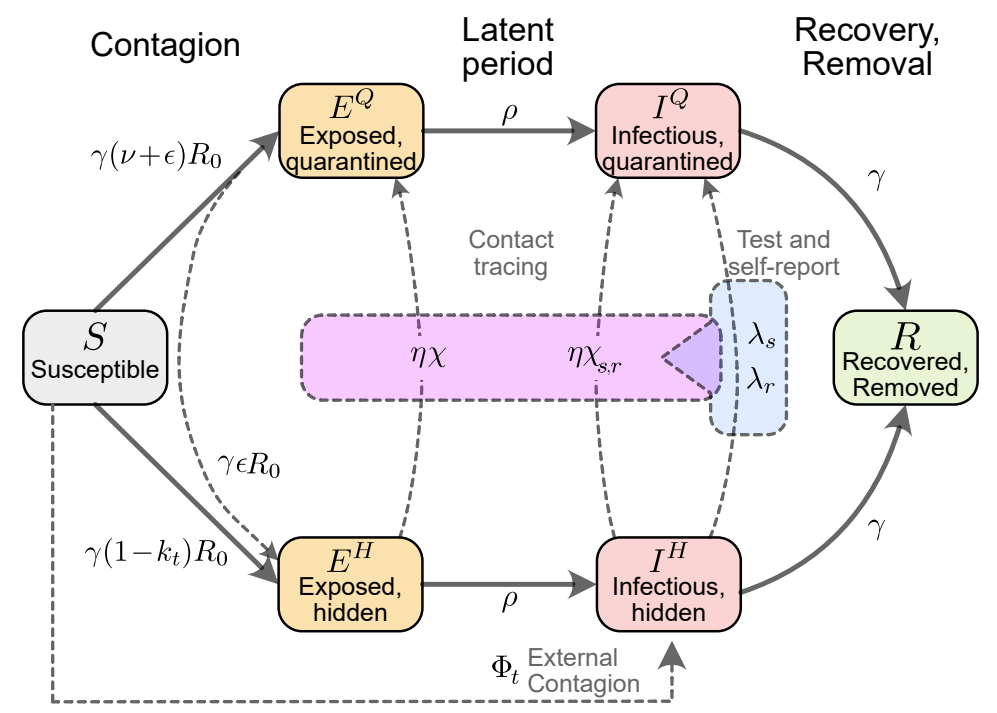

Supplementary Figure S2: Flowchart of the complete model.

reached - assuming the duration of individual immunity is long enough. That high level of new infections leads to a considerable death toll, as currently about $1.5 \%$ of the infected individuals would die (depending on age structure $\left.{ }^{[7 ;[\mathrm{G7}}\right)$. Moreover, containment measures like quarantine become unsustainable because, if implemented, each one of the 200 daily new infected cases would require the quarantine of 5-50 people (their high-risk contacts) for about 10 days, causing $1-10 \%$ of the population being in quarantine at any given day. Therefore the alleged economical and social benefits of such a strategy ${ }^{\mathbf{6 0 ;} ; \mathbf{6 9}}$ may be questionable.

\section{S2.2 Linear stability analysis and uncertainty propagation}

For analysing the stability of the governing differential equations, namely, whether an outbreak could be controlled, we studied the linear stability of the system. Moreover, we consider that, within the timeframe considered for stability purposes, the fraction $\frac{S}{M}$ would remain somewhat constant, we consider the

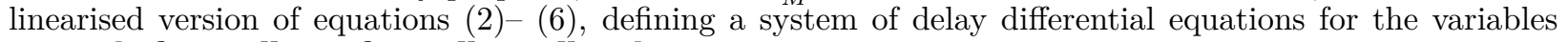
$x(t)=\left[E^{Q}(t) ; E^{H}(t) ; I^{Q}(t) ; I^{H}(t) ; I^{H, s}(t)\right]$. We define matrices $A$ and $B$ as:

$$
\begin{aligned}
A= & \left(\begin{array}{ccccc}
-\rho & 0 & \nu \gamma R_{0} & 0 & 0 \\
0 & -\rho & \epsilon \gamma R_{0} & \gamma\left(1-k_{t}\right) R_{0} & 0 \\
\rho & 0 & -\gamma & \lambda_{r} & \lambda_{s} \\
0 & \rho & 0 & -\gamma-\lambda_{r} & -\lambda_{s} \\
0 & (1-\xi) \rho & 0 & 0 & -\gamma-\lambda_{r}-\lambda_{s}
\end{array}\right) \\
B= & \left(\begin{array}{cccccc}
0 & 0 & 0 & \lambda_{r}^{\mathrm{eff}} \chi_{\tau} & \lambda_{s}^{\mathrm{eff}} \chi_{\tau} \\
0 & 0 & 0 & -\lambda_{r}^{\mathrm{eff}} \chi_{\tau} & -\lambda_{s}^{\mathrm{eff}} \chi_{\tau} \\
0 & 0 & 0 & \lambda_{r}^{\mathrm{eff}}\left(\xi \chi_{r}+(1-\xi) \chi_{s, r}\right) & \lambda_{s}^{\mathrm{eff}}\left(\xi \chi_{r}+(1-\xi) \chi_{s, r}\right) \\
0 & 0 & 0 & -\lambda_{r}^{\mathrm{eff}}\left(\xi \chi_{r}+(1-\xi) \chi_{s, r}\right) & -\lambda_{s}^{\mathrm{eff}}\left(\xi \chi_{r}+(1-\xi) \chi_{s, r}\right) \\
0 & 0 & 0 & -\lambda_{r}^{\mathrm{eff}}(1-\xi) \chi_{s, r} & -\lambda_{s}^{\mathrm{eff}}(1-\xi) \chi_{s, r}
\end{array}\right) \eta\left(1-k_{t}\right) R_{0},
\end{aligned}
$$

where

$$
\lambda_{r}^{\mathrm{eff}}=\frac{\gamma \lambda_{r}}{\lambda_{r}+\gamma}, \quad \lambda_{s}^{\mathrm{eff}}=\gamma\left(\frac{\lambda_{s}+\lambda_{r}}{\gamma+\lambda_{s}+\lambda_{r}}-\frac{\lambda_{r}}{\lambda_{r}+\gamma}\right) .
$$

The equations governing the dynamics for vector $x(t)$ are then presented in their matrix form:

$$
x^{\prime}(t)=A x(t)+B x(t-\tau) .
$$




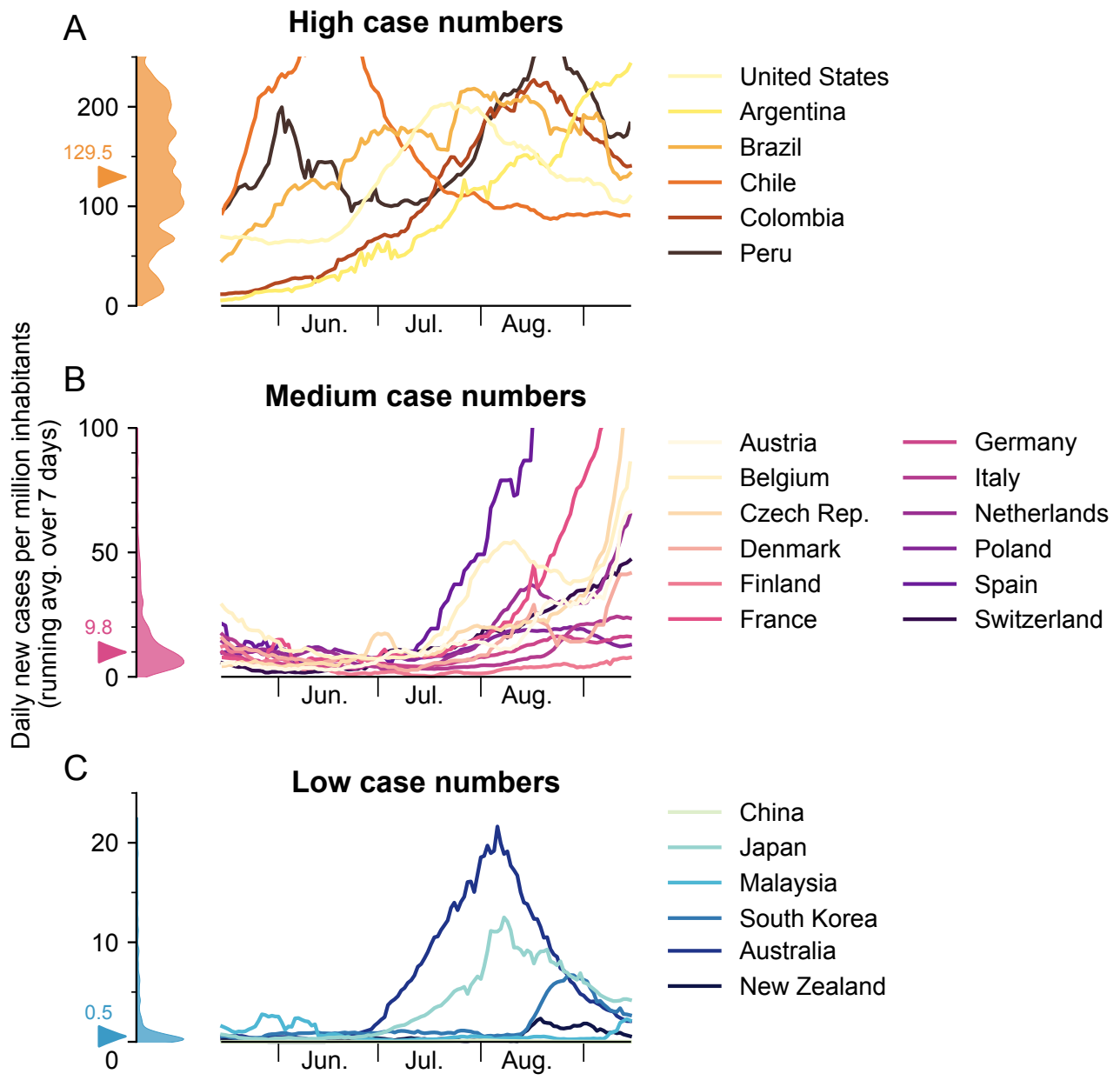

Supplementary Figure S3: Strategies of countries to fight SARS-CoV-2 differ widely and are reflected in case numbers. (A) Strategies that involve little nonpharmaceutical interventions rely on the population to hinder the spread in a self-regulated manner and are often accompanied by high case numbers. Examples can be dominantly found in North- and South-America, the median of daily new cases for the shown examples and time range exceeds 100 per million inhabitants per day. (B) Strategies that aim to keep case numbers low through extensive TTI, combined with temporary lockdowns can lead to medium case numbers. As the effectiveness of TTI measures depends on daily infections, case numbers can seemingly explode when the (hard-to-estimate) TTI capacity limit is exceeded. Many European countries managed to stabilise case numbers over the summer but are now seeing case numbers that rise faster-than-exponential. The median of daily new cases for the shown examples and time range is 9.88 per million per day. (C) When the external influx is low and/or employed strategies to reduce contacts are very effective, the stable regime can be reached. In this case, average case numbers are very low and local outbreaks can be controlled well through local interventions. Examples can be dominantly found in East-Asia and Oceania. The median of daily new cases for the shown examples and time range is 0.55 per million per day. Raw data and preliminary visualizations were obtained from ${ }^{44}$.

We determine the critical values for the contact reduction $k_{t}^{\text {crit }}$, for which exponential solutions would be asymptotically stable. Eigenvalues were determined by systematically solving the nonlinear eigenvalues problem for stability ${ }^{[\mathrm{g}}$, where the solution operation was approximated with a Chebyshev differentiation matrix ${ }^{60}$. Eigenvalues, in this sense, would be solutions of the scalar equation

$$
\operatorname{det}\left(-s I+A+e^{-s \tau} B\right)=0
$$

Noting that $A$ and $B$ explicitly depend on the model parameters, we numerically explore which combinations of them would result in stable, metastable or unstable case numbers. Concretely, we studied the minimum -critical- contact reduction required for stablilization $k_{t}^{\text {crit }}$ in two scenarios; i) low case numbers, and TTI 
medRxiv preprint doi: https://doi.org/10.1101/2020.12.10.20247023; this version posted December 11, 2020. The copyright holder for this preprint (which was not certified by peer review) is the author/funder, who has granted medRxiv a license to display the preprint in perpetuity. All rights reserved. No reuse allowed without permission.

Low case numbers enable long-term stable pandemic control without lockdowns

fully operative (both testing and contact tracing), and ii) high case numbers, above the TTI limit, where testing would be inefficient and solely symptom driven $\left(\lambda_{s}=\lambda_{s}^{\prime}, \eta=\lambda_{r}=\lambda_{r}^{\prime}=0\right)$.

To explore the uni-variate impact different signature parameters have on $k_{t}^{\text {crit }}$, we studied the zeros of ([E]) as a function of $k_{t}$ using the @f zero MATLAB function (Fig. S4AA). Using the same routines and a random sampling procedure, we propagate uncertainties in the values of these parameters, uni-variate (Fig. S4B), and multivariate (Fig. S4IC).

Supplementary Table S3: Parameter uncertainty propagation

\begin{tabular}{|c|c|c|c|c|c|c|c|}
\hline Parameter & Meaning & Median & $95 \%$ CI & $\alpha$ & $\beta$ & Dist. & Units \\
\hline$\xi$ & Asymptomatic ratio & 0.32 & $0.23-0.42$ & 27.5 & 27.8 & beta & - \\
\hline$\lambda_{s}$ & Symptom-driven test rate & 0.25 & $0.20-0.31$ & 56 & 168 & beta & days $^{-1}$ \\
\hline$\nu$ & $\begin{array}{l}\text { Registered contacts (quaran- } \\
\text { tined) }\end{array}$ & 0.07 & $0.03-0.13$ & 8.25 & 101.8 & beta & - \\
\hline$\eta$ & Tracing efficiency & 0.66 & $0.59-0.73$ & 117.9 & 60.7 & beta & - \\
\hline$\epsilon$ & Lost contacts (quarantined) & 0.05 & $0.01-0.11$ & 3.8 & 71.25 & beta & - \\
\hline$\left.k_{t}^{\text {crit }}\right|_{\text {TTI }}$ & $\begin{array}{l}\text { Necessary contact reduction } \\
\text { with TTI }\end{array}$ & $39 \%$ & $24-53 \%$ & - & - & - & - \\
\hline$\left.k_{t}^{\text {crit }}\right|_{\text {no TTI }}$ & $\begin{array}{l}\text { Necessary contact reduction } \\
\text { without TTI }\end{array}$ & $58 \%$ & $53-62 \%$ & - & - & - & - \\
\hline
\end{tabular}

Supplementary Table S4: Linearly-derived correspondence between contact reduction and the observed reproduction number

\begin{tabular}{lll}
\hline$k_{t}$ & $\hat{R}_{t}^{\text {obs }}$ (with TTI) & $\hat{R}_{t}^{\text {obs }}$ (without TTI) \\
\hline 0.2 & 1.08 & 1.26 \\
0.4 & 0.99 & 1.13 \\
0.6 & 0.91 & 0.98 \\
0.75 & 0.86 & 0.88 \\
\hline
\end{tabular}




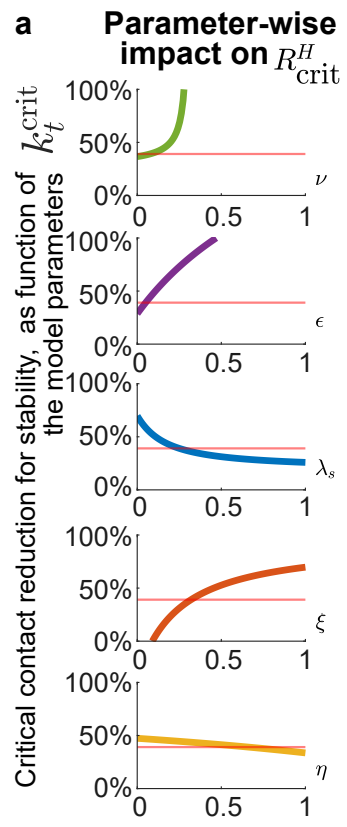

b Parameter-wise propagation of uncertainty
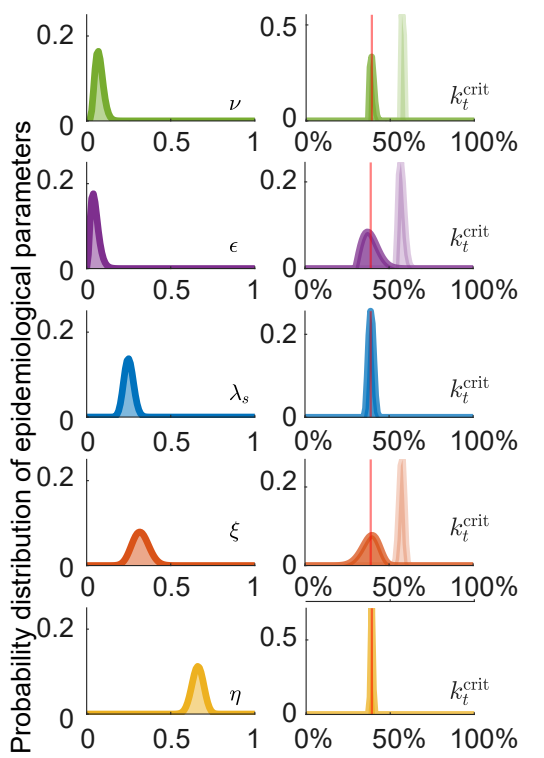

C
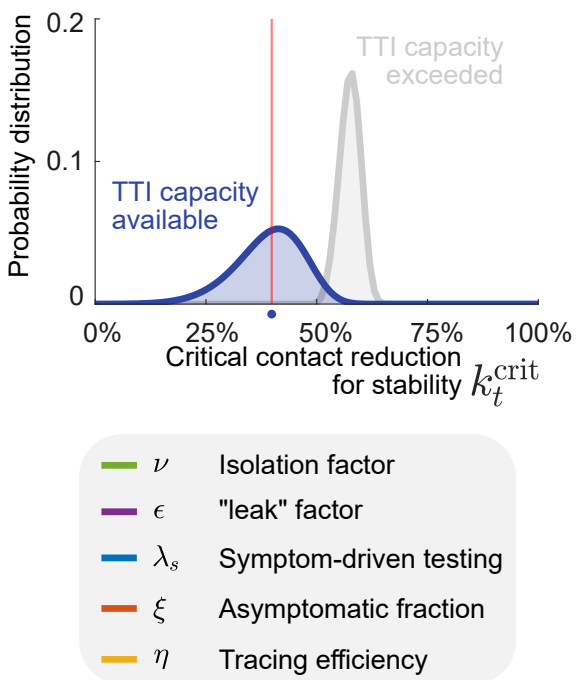

Supplementary Figure S4: Propagation of TTI-parameter uncertainties to the critical contact reduction required for stability. As the different parameters involved in our model play different roles, the way their variability propagates to $k_{t}^{\text {crit }}$ differs, even when their variability profiles look similar. a: Impact of single-parameter variation on the critical hidden reproduction number $k_{t}^{\text {crit }}$. To evaluate the monotony (direction) of their impact on $k_{t}^{\text {crit }}$, we scan their entire definition range, ignoring the practical feasibility of achieving such values. Dotted black line shows the default critical hidden reproduction number. b: Univariate uncertainties of TTI parameters modelled by beta distributions centred on their default value, and the resulting distribution of critical reproduction numbers $k_{t}^{\text {crit }}$ (right column). Results are shown assuming testing only (light colours) or testing and tracing (dark colours). The default value of $k_{t}^{\text {crit }}$ is marked by the dotted lines, in the presence (black) or absence (grey) of tracing. c: Distribution of critical reproduction numbers arising from multivariate uncertainty propagation given by the joint of the distributions shown in (A) for testing only (light colours), or testing and tracing (dark colours). The default value of $R_{\text {crit }}^{H}$ is marked by the dotted lines, in the presence (black) or absence (grey) of tracing. Results show averages of 100000 realisations.

\section{S2.3 On the contact reduction required for achieving early herd immunity.}

In Supplementary Section 52.2, we derived a methodology for obtaining the minimal, critical contact reduction $k_{t}^{\text {crit }}$ for which the linear system is asymptotically stable. Such values, however, assume a fully susceptible population, as we ignore the scaling factor $\frac{S}{M}$.

The herd immunity threshold $\varrho$ represents the fraction of the population that needs to be immunised for controlling the spread of an infectious disease. It can be expressed in terms of the effective reproduction number $R_{t}$ :

$$
\varrho=1-\frac{1}{R_{t}} .
$$

In the context of our model, $R_{t}$ can be expressed in terms of the reduction of contagious contacts $k_{t}$ and the basic reproduction number $R_{0} ; R_{t}=\left(1-k_{t}\right) R_{0}$. Nonetheless, in further stages of an ongoing outbreak, the fraction of people that is no longer susceptible would have an effect, thus we include also the scaling factor $\frac{S}{M}$ :

$$
R_{t}=\left(1-k_{t}\right) \frac{S}{M} R_{0}
$$


Combining both equation $(\mathbb{Z Z})$ and $(\mathbb{Z Z})$, we can express $\varrho$ as

$$
\varrho=1-\frac{1}{\left(1-k_{t}\right)(1-f) R_{0}}
$$

assuming a quasi-stationary dynamics for $S$, and defining $f=1-\frac{S}{M}$. Suppose we study the case in which no major behavioural changes take place, thus the herd immunity threshold $\varrho$ would remain the same. On the other hand, because of the sole fact of having a progressively increasing immunisation among the population (because of vaccination or post-infection immunity), the required reduction of contacts for stabilisation $k_{t}^{\text {crit }}$ will decrease. Assuming critical conditions, we use $k_{t}=k_{t}^{\text {crit }}$ in equation (区.M):

$$
\varrho=1-\frac{1}{\left(1-k_{t}^{\text {crit }}\right)(1-f) R_{0}}
$$

As we assumed that no behavioural change is taking place, we obtain the herd immunity threshold by only evaluating equation $(\mathbb{E Z})$ at $f=0$.

$$
\varrho=1-\frac{1}{\left(1-k^{\mathrm{crit}, 0}\right) R_{0}},
$$

and subtracting (Bत) and (Bत) we obtain an expression for $k_{t}^{\text {crit }}(f)$

$$
k_{t}^{\mathrm{crit}}(f)=1-\frac{1-k^{\mathrm{crit}, 0}}{1-f}
$$

\section{S2.4 Inferring the reproduction number of COVID-19 from Jun to Oct. 2020}

We use the framework of Bayesian inference on an SIR model presented in our previous work ${ }^{\mathbb{1 1}}$ to infer the daily growth rate $\lambda^{*}(t)$. An SIR compartmental model with weekly change points is used whose central epidemiological parameters are inferred using the PyMC3 package ${ }^{\text {II }}$. A weekly modulation was applied to take weekly reporting structure and weekend delays into account. After inference a rolling average was performed on the daily case numbers for comparability and clarity. The effective reproduction number $\hat{R}_{t}^{\text {eff }}$ can be expressed dependent on the effective daily growth rate:

$$
\hat{R}_{t}^{\mathrm{eff}}=\left(\lambda^{*}(t)+1\right)^{4}
$$

This short analysis was performed for Germany (Fig. 55.5) and other European countries (Fig. 56]) which showed the same metastable behaviour, while their case numbers were below a threshold around 50 cases per day (per million). A transition into the unstable regime can be seen once case numbers surpass this threshold.

\section{S2.5 On the incorporation of random testing in the TTI scheme}

Testing can also be done in a random manner or randomly combined with a tracing strategy. Even though the amount of tests that would be required for such purposes would be enormous, the development of fast, cheap, and reliable tests offers an interesting alternative to consider.

In this supplementary note we derive the equations presented in the methods but also including random testing. As therein described, random testing is assumed to occur at a constant rate $\lambda_{r}$, which, for default parameters, reflect the amount of tests per day per million people. In that way, it seems reasonable to consider unfeasible testing rates surpassing $\lambda_{r}^{\max }=0.1$, as it would mean that a $10 \%$ of the population is tested everyday. 


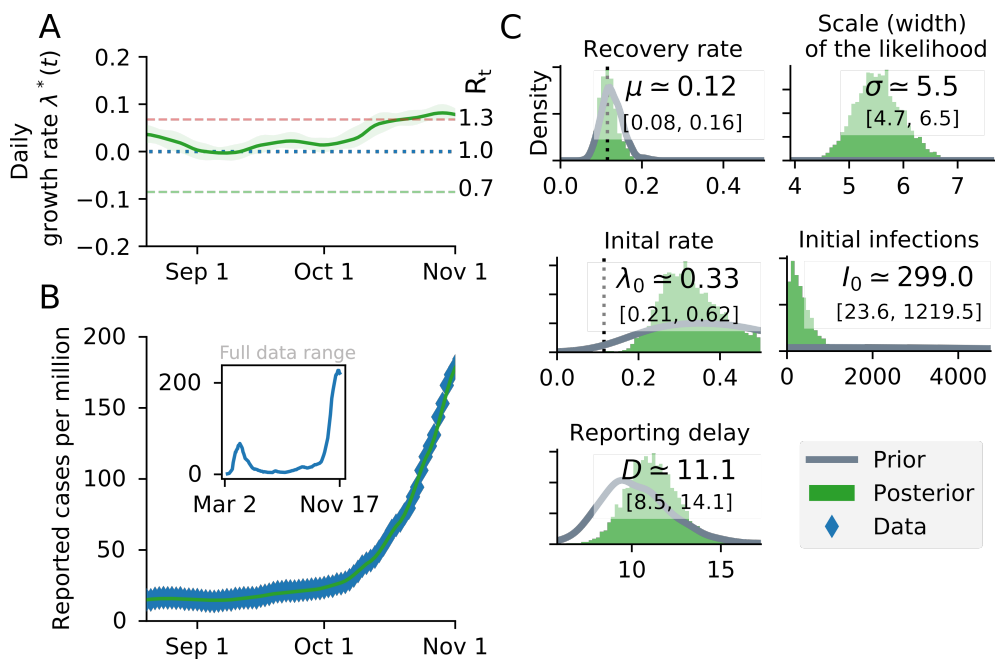

Supplementary Figure S5: A substantial increase in the effective growth rate in Germany occurred during the month of October, suggesting that regional TTI capacity limits were exceeded. (A) Before October, the reproduction number was slightly above one (corresponding to a daily growth rate $\lambda^{*}$ slightly above zero). (B) Observed case numbers were stabilised below 20 daily new cases per million (but still, slowly growing), until a transition into the unstable regime took place over $\sim 4$ weeks in October. The time range is adjusted to focus on this tipping point. The inset shows case numbers for the full available time range. (C) Main central epidemiological parameters with prior and posterior distribution.

\section{S2.5.1 Number of cases observable through testing $N^{\text {test }}$}

When random testing is included in the scheme, the solution of equation (ㅁ]) for the symptomatic and asymptomatic infections -hidden- would be given by equations (34) and (3.5).

$$
\begin{aligned}
& \chi_{s, r}=\left\{\begin{array}{l}
\rho \tau \exp (-\rho \tau), \quad \text { if } \rho \approx \lambda_{s}+\lambda_{r} \\
\frac{\rho}{\left(\lambda_{s}+\lambda_{r}\right)-\rho}\left(\exp (-\rho \tau)-\exp \left(-\tau\left(\lambda_{s}+\lambda_{r}\right)\right)\right), \text { else. }
\end{array}\right. \\
& \chi_{r}=\left\{\begin{array}{l}
\rho \tau \exp (-\rho \tau), \quad \text { if } \rho \approx \lambda_{r} \\
\frac{\rho}{\lambda_{r}-\rho}\left(\exp (-\rho \tau)-\exp \left(-\tau \lambda_{r}\right)\right), \text { else. }
\end{array}\right.
\end{aligned}
$$

If both symptom-based and random testing take place simultaneously, the number of discovered infections is given by

$$
N^{\text {test }}=\lambda_{r} I^{H}+\lambda_{s} I^{H, s}
$$

Further, assuming that after reaching $N_{\max }^{\text {test }}$, the testing rates at the overhead pool-sizes would decrease to $\lambda_{s}^{\prime}$ and $\lambda_{r}^{\prime}$, respectively, for symptom-driven and random testing. We further assume that testing resources would be exclusively allocated to sustain the symptom-driven testing in our default scenario. The overall testing term $N^{\text {test }}$ would be given by:

$$
\begin{aligned}
N^{\text {test }}=\lambda_{r} \min \left(I^{H}, I_{\max }^{H}\right) & +\lambda_{r}^{\prime} \max \left(0, I^{H}-I_{\max }^{H}\right) \\
& +\lambda_{s} \min \left(I^{H, s}, I_{\max }^{H, s}\right)+\lambda_{s}^{\prime} \max \left(0, I^{H, s}-I_{\max }^{H, s}\right),
\end{aligned}
$$

where $I_{\max }^{H, s}, I_{\max }^{H}$ represent the pool sizes of the symptomatic hidden and total hidden pools, respectively, at the TTI limit, i.e. $\lambda_{r} I_{\max }^{H}+\lambda_{s} I_{\max }^{H, s} \stackrel{!}{=} N_{\max }^{\text {test }}$, reached at time $t=t^{*}$. Defining $\varphi:=\left.\frac{I^{H, s}}{I^{H}}\right|_{t=t^{*}}$, we can express such magnitudes in term of the maximum capacity $N_{\max }^{\text {test }}$ : 
medRxiv preprint doi: https://doi.org/10.1101/2020.12.10.20247023; this version posted December 11, 2020. The copyright holder for this preprint (which was not certified by peer review) is the author/funder, who has granted medRxiv a license to display the preprint in perpetuity. All rights reserved. No reuse allowed without permission.

Low case numbers enable long-term stable pandemic control without lockdowns

Belgium

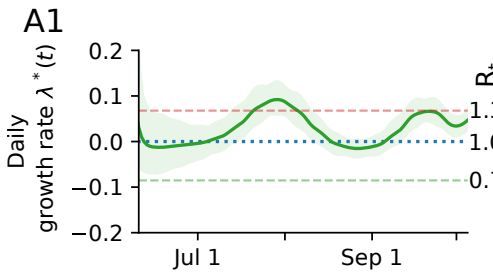

A2

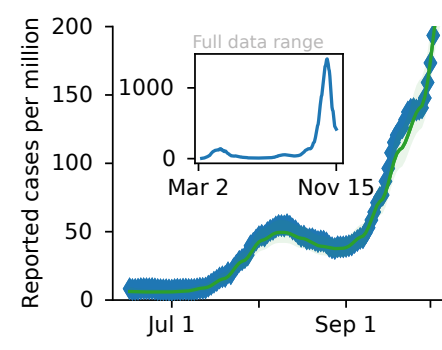

Poland

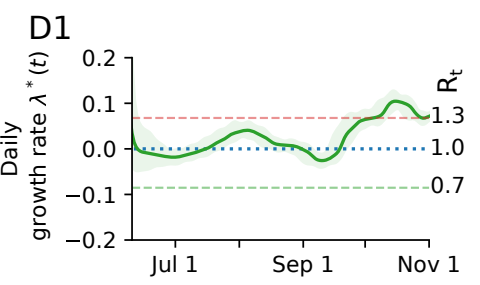

D2

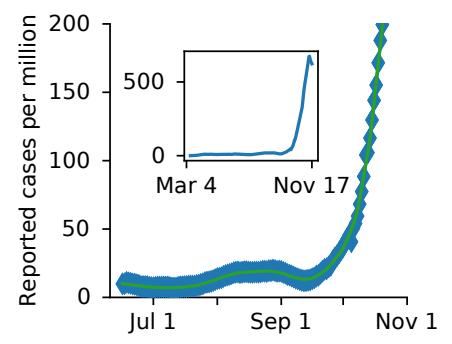

Greece

B1

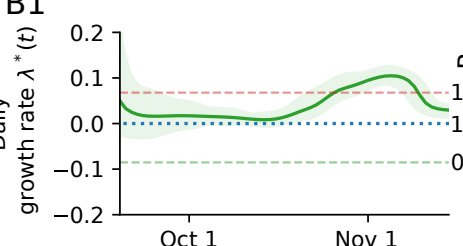

B2

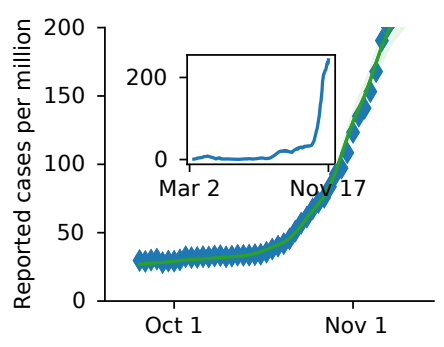

Portugal

E1

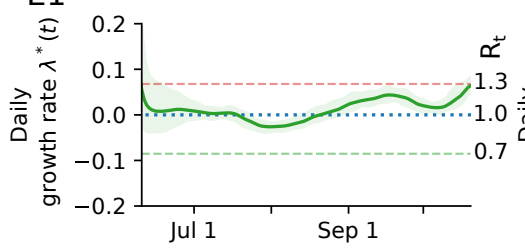

E2

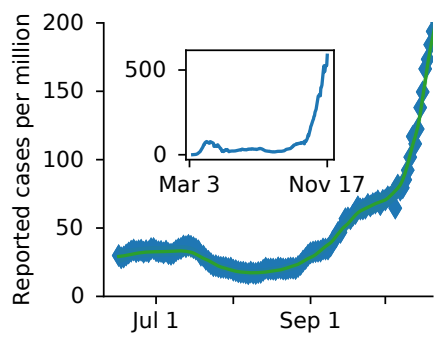

Italy

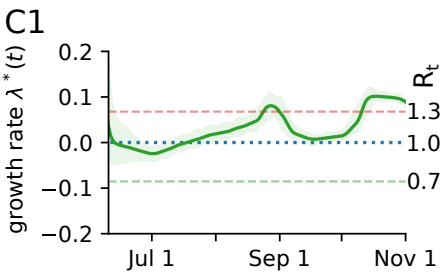

C2

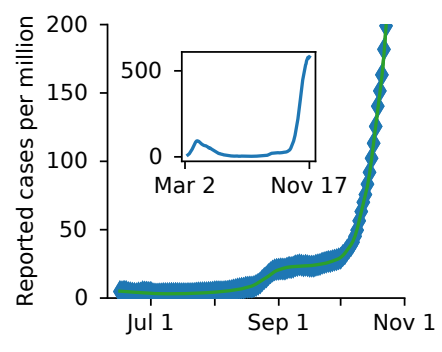

Switzerland

F1

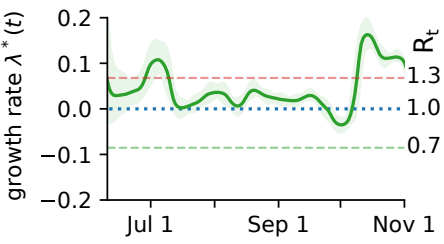

F2

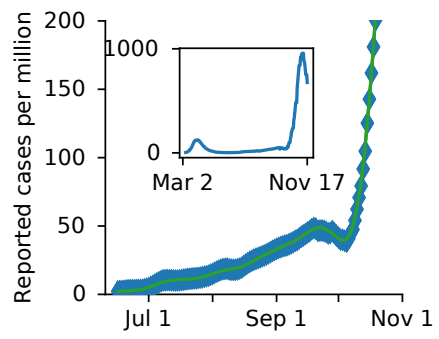

Supplementary Figure S6: Comparison of the reproduction number and reported cases as second wave emerges in different European countries. For each country, parameters of an SIR model were fitted to the reported data of the Our World in Data repository ${ }^{\sqrt{44}}$, following the procedure presented in ${ }^{[1 \times}$. (Panels X1) The time-dependent effective growth rate stays between -0.1 and 0.1 and rises before the tipping point. This corresponds to an effective reproduction number between 0.7 and 1.3 , which matches our preliminary assumptions. The time range is adjusted to focus on the tipping point. (Panels X2) After a (meta-)stable regime in summer, all of the selected countries show a rise in case numbers and a tipping point at around 50 new cases per day per million, where the spread self-accelerates and the cases increase significantly. (Insets) Case numbers for the full available time range.

$$
\begin{aligned}
I_{\max }^{H, s} & =\frac{\varphi N_{\max }^{\mathrm{test}}}{\varphi \lambda_{s}+\lambda_{r}} \\
I_{\max }^{H} & =\frac{N_{\max }^{\mathrm{test}}}{\varphi \lambda_{s}+\lambda_{r}} .
\end{aligned}
$$


medRxiv preprint doi: https://doi.org/10.1101/2020.12.10.20247023; this version posted December 11, 2020. The copyright holder for this preprint (which was not certified by peer review) is the author/funder, who has granted medRxiv a license to display the preprint in perpetuity. All rights reserved. No reuse allowed without permission.

Low case numbers enable long-term stable pandemic control without lockdowns

The explicit value of $\varphi$ can be obtained numerically in the integration routine, or estimated trough the use of the equilibrium values of the differential equations, $\varphi=\frac{I_{\infty}^{H, s}}{I_{\infty}^{H}}$ (as implemented in our code). The expression for the symptomatic hidden pool $I^{H, s}$ in the presence of random testing is slightly different;

$$
\begin{aligned}
N_{s}^{\mathrm{test}}=\lambda_{r} \min \left(I^{H, s}, I_{\max }^{H, s}\right) & +\lambda_{r}^{\prime} \max \left(0, I^{H, s}-I_{\max }^{H, s}\right) \\
& +\lambda_{s} \min \left(I^{H, s}, I_{\max }^{H, s}\right)+\lambda_{s}^{\prime} \max \left(0, I^{H, s}-I_{\max }^{H, s}\right) .
\end{aligned}
$$

\section{S2.5.2 Random testing: Number of cases observable through contact-tracing $N^{\text {traced }}$}

Because of TTI, infectious individuals move (or are likely to move) from the hidden to the quarantined infectious pool before recovering. Therefore, they spend a comparatively shorter amount of time there and, on average, would not generate the expected amount of offspring infections as some would be prevented. Assuming the average residence time of untested individuals is $\frac{1}{\gamma}$, we estimate the residence time of individuals removed by symptom-driven testing and random testing. Noting that symptomatic individuals can be tested and therefore removed by any of the testing criteria, their residence time would be approximately $\frac{1}{\gamma+\lambda_{s}+\lambda_{r}}$, whereas the average residence time of asymptomatic individuals would be $\frac{1}{\gamma+\lambda_{r}}$. Therefore, the fractions of time that symptomatic and asymptomatic individuals stay unnoticed are respectively

$$
t_{s}=\frac{\gamma}{\gamma+\lambda_{s}+\lambda_{r}}
$$

and

$$
t_{r}=\frac{\gamma}{\gamma+\lambda_{r}}
$$

If the daily new cases observed through testing, delayed at the moment of processing, $N_{t-\tau}^{\text {test }}$, are within the tracing capacity of the health authorities, i.e. $N_{t-\tau}^{\text {test }} \leq N_{\max }^{\text {test }}$, then $N^{\text {traced }}$ is defined as

$$
N^{\text {traced }}=\eta R_{t-\tau}^{H}\left(I_{t-\tau}^{H} t_{r} \lambda_{r}+I_{t-\tau}^{H, s}\left(t_{s} \lambda_{s}+\left(t_{s}-t_{r}\right) \lambda_{r}\right)\right) .
$$

Otherwise, using the expressions for $I^{H}$ and $I^{H, s}$ when the TTI capacity is reached derived in the previous section, we can obtain an effective rate

$$
\lambda_{\mathrm{eq}}=\frac{\lambda_{r}(1-\varphi) t_{r}+\varphi \lambda_{s} t_{s}}{\lambda_{r}+\varphi \lambda_{s}} .
$$

Therefore, the average amount of positive cases identified by contact tracing in the TTI limit is given by

$$
N^{\text {traced }}=\eta R_{t-\tau}^{H} N_{\max }^{\text {test }} \lambda_{\text {eq }}
$$

To sum up the last equations:

$$
N^{\text {traced }}= \begin{cases}\eta R_{t-\tau}^{H}\left(I_{t-\tau}^{H} t_{r} \lambda_{r}+I_{t-\tau}^{H, s}\left(t_{s} \lambda_{s}+\left(t_{s}-t_{r}\right) \lambda_{r}\right)\right) & \text { if } N_{t-\tau}^{\text {test }} \leq N_{\max }^{\text {test }} \\ \eta R_{t-\tau}^{H} N_{\max }^{\text {test }} \lambda_{\text {eq }} & \text { else }\end{cases}
$$

